\title{
Polarimetric SAR Change Detection with the Complex Hotelling-Lawley Trace Statistic
}

\author{
Vahid Akbari, Member, IEEE, Stian N. Anfinsen, Member, IEEE, Anthony P. Doulgeris, Member, IEEE \\ Torbjørn Eltoft, Member, IEEE, Gabriele Moser, Member, IEEE, and Sebastiano B. Serpico, Fellow, IEEE
}

\begin{abstract}
In this paper, we propose a new test statistic for unsupervised change detection in polarimetric radar images. We work with multilook complex covariance matrix data, whose underlying model is assumed to be the scaled complex Wishart distribution. We use the complex-kind Hotelling-Lawley trace statistic for measuring the similarity of two covariance matrices. The distribution of the Hotelling-Lawley trace statistic is approximated by a Fisher-Snedecor distribution, which is used to define the significance level of a false alarm rate regulated change detector. Experiments on simulated and real PolSAR data sets demonstrate that the proposed change detection method gives detections rates and error rates that are comparable with the generalized likelihood ratio test.
\end{abstract}

Index Terms-Synthetic aperture radar, polarimetry, Hotelling-Lawley trace statistic, likelihood ratio test statistic, Fisher-Snedecor distribution, complex Wishart distribution, change detection.

\section{INTRODUCTION}

$\mathbf{M}$ ULTITEMPORAL remote sensing represents a powerful source of information to monitor and study processes on the Earth's surface, for instance, in detection and monitoring of volcanic activity, disaster management, and monitoring of glaciers and urban growth. The use of synthetic aperture radar (SAR) sensors is attractive in temporal studies, because SAR sensors do not suffer from the limitations of cloud cover and solar incidence, contrary to optical sensors. Many studies have demonstrated the potential of SAR images in change detection and time series analysis, e.g., [1]-[6]. This study is devoted to the multichannel polarimetric SAR (PolSAR) sensor, which potentially provides increased detection capability, as compared to single-polarization SAR [7].

Unsupervised change detection in SAR images is generally performed in three sequential steps: 1) image preprocessing, 2) computing a test statistic, and 3) making a decision based on analysis of the test statistic computed in step 2 [8]. Preprocessing includes coregistration, geometric and radiometric corrections, and noise reduction. In the second step, two preprocessed images are combined by a desired test statistic which can be used in step 3 to test the hypotheses

Manuscript received March 31, 2015; revised October 02, 2015 and January 11, 2015; accepted Febuary 17, 2016.

V. Akbari, A. P. Doulgeris, T. Eltoft, and S. N. Anfinsen are with the Department of Physics and Technology, University of Troms $\emptyset$, 9037 Troms $\emptyset$, Norway (e-mail:va2akbari@gmail.com;anthony.p.doulgeris @uit.no;torbjorn.eltoft@uit.no;stian.normann.anfinsen@uit.no).

G. Moser and S. B. Serpico are with the Department of Electrical, Electronic, Telecommunications Engineering, and Naval Architecture, University of Genoa, 16145 Genoa, Italy (e-mail:gabriele.moser@unige.it; sebastiano.serpico@unige.it). of change versus no-change. As an example, the ratio of SAR amplitudes or intensities observed at different times is a well-known test statistic in single-channel SAR-based change detection. Similarly, image differencing and change vector analysis are classical approaches to change detection with optical images. In the analysis of multitemporal single-channel SAR images, a large number of test statistics have been developed and described in the literature for automatic and unsupervised change detection, such as image ratioing [3], [9], mean-ratio detector [10], log-ratio operator [2], multitemporal coherence analysis [1], fuzzy hidden Markov chains [11], postclassification comparision change detection [12], maximumlikelihood ratio [13], and Kullback-Leibler (KL) divergence [4].

The generalization of these methods to multichannel SAR images has proved nontrivial. Hence, the literature on change detection with PolSAR data is more sparse. We limit our focus to change detection in multilook complex (MLC) PolSAR images, where the backscattered signal is represented by the so-called polarimetric sample covariance (or coherency) matrix. For each pixel, this is a Hermitian and positive definite matrix, which is often assumed to follow a complex Wishart distribution. The seminal work on test statistics for change detection in multilook PolSAR images was done by Conradsen et al., who proposed a generalized likelihood ratio test (LRT) for equality of two complex covariance matrices and gave the asymptotic sampling distribution for the test statistic [14]. This method, also known as the Bartlett test, was compared by Kersten et al. to the alternative contrast ratio and ellipticity tests [15]. Erten et al. presented a new coherent similarity measure for multitemporal multichannel SAR images by means of mutual information [16]. Marino et al. proposed a test which is reported to suppress intensity information and perform well in detection of changes in the internal structure of the covariance matrix [17]. All of the mentioned approaches assume that the covariance matrices follow the complex Wishart distribution.

A new similarity measure for change detection in nonWishart PolSAR data described by the multilook product model was recently presented by Liu et al. [18]. Their model flexibility comes at the expense of higher computational cost, since they use a test statistic which contains the Bessel $\mathrm{K}$ function and requires estimation of the shape parameter of the model. A simpler approach which still makes allowance for radar texture is proposed in [19]. Other methods proposed recently include extensions of the LRT to multitemporal [20] and multifrequency data [21], a patch based change detector 
which works on speckle filtered data [22], other objectoriented methods utilizing post-classification comparison [23], [24], and an approach to processing of time series of PolSAR data [25]. In this paper, we propose a simpler test statistic which still assumes the complex Wishart distribution for the covariance matrix data, and yet is able to detect changes in many scenarios.

In the third step of the change detection analysis, changes are detected by applying a decision threshold to the test statistic. Several algorithms have been proposed to determine the threshold in a completely unsupervised manner, such as the constant false alam rate (CFAR) algorithm [26] (whose false alarm regulation principle is used also when the CFAR property does not hold), Otsu's method [27], the Kittler and Illingworth (K\&I) algorithm [28], the entropy-based Kapur algorithm [29], and Liu's method [30].

In this study, we apply the complex-kind Hotelling-Lawley trace (HLT) [31] as a new test statistic for MLC PolSAR data. The method can be seen as a matrix-variate version of the intensity ratio test, where the HLT is used to contrast two complex covariance matrices and produce a scalar value, to which a threshold can be applied. Note that both low and high values of the test statistic indicate change, by analogy with the intensity ratio. The sampling distribution of the proposed test statistic has been approximated by the FisherSnedecor (FS) distribution. This is motivated by previous use of this distribution as an approximate model for the real-kind Hotelling-Lawley trace [32]-[34].

The parameters of the FS distribution are determined by matching population moments of the HLT and the FS distribution, and depend only on the dimension of the polarimetric data and the equivalent number of looks (ENL) estimated for the images. Eventually, a binary decision can be made to get a final change map at a predefined false alarm rate (FAR). We compare the change detection results obtained from our proposed method with the complex Wishart-based LRT statistic proposed in [14]. Simulated PolSAR images generated with complex Wishart distribution matrix data are used for this comparison with focus on areas where different types of change are imposed.

A critical part of the proposed detection algorithm is the estimation of the ENL. In multilook processing, the averaged pixels become correlated and this complicates the statistical modeling of the test statistic. The usual solution is to replace the actual number of correlated samples used in the multilook averaging with an noninteger number of independent samples that would hold the same amount of information. We incorporate the estimation technique from [35], where the ENL is estimated using the method of matrix log-cumulants in a preanalysis of the whole image. This method is automatic and avoids the alternative approach of identifying a large area with homogeneous reflectivity, which is often not feasible.

The remainder of this paper is organized as follows. In Section II, we describe the polarimetric SAR data and the model for the covariance matrix data. In Section III, the proposed change detection algorithm is explained, followed by the ENL estimation, the proposed test statistic, its sampling distribution, and CFAR-type thresholding. In Section IV, we briefly discuss the method proposed by Conradsen $e t$ al., which is to be compared with our method in this study. Section V demonstrates the performance of the method with a simulated PolSAR data set and two RADARSAT-2 fully polarimetric images of Suzhou, China and discusses the results. Section VI is dedicated to a summary and conclusions.

\section{Polarimetric SAR Image Model}

A full-polarimetric imaging radar measures the amplitude and phase of backscattered signals in the four combinations of the linear receive and transmit polarizations: $\mathrm{HH}, \mathrm{HV}, \mathrm{VH}$, and VV. Assuming that the target reciprocity condition is satisfied [36], the backscattering of a monostatic polarimetric SAR system is characterized by the complex scattering vector, $\Omega$, with dimension $d=3$, as given by

$$
\boldsymbol{\Omega}=\left[S_{h h}, \sqrt{2} S_{h v}, S_{v v}\right]^{T} \in \mathbb{C}^{3} .
$$

The elements represent the complex backscattering coefficients in three polarimetric channels, noting that $S_{h v}$ is a coherent average of the $\mathrm{HV}$ and $\mathrm{VH}$ channel measurements. The superscript $T$ denotes the matrix transpose, and $\sqrt{2}$ arises from the requirement to conserve the total scattered power, after coherent averaging of the cross-polarization channels.

The vector $\Omega$ is a single-look complex (SLC) format representation of polarimetric SAR data. Single and dual-channel polarimetric data can be treated in a similar way, as subsets of lesser dimension and most likely with less information. The scattering vectors are transformed into multilooked sample covariance matrices in order to reduce speckle noise, at the expense of spatial resolution, i.e.,

$$
\begin{aligned}
\mathbf{C} & =\frac{1}{L} \sum_{\ell=1}^{L} \boldsymbol{\Omega}_{\ell} \boldsymbol{\Omega}_{\ell}^{H} \\
& =\left[\begin{array}{ccc}
\left\langle\left|S_{h h}\right|^{2}\right\rangle & \left\langle\sqrt{2} S_{h h} S_{h v}^{*}\right\rangle & \left\langle S_{h h} S_{v v}^{*}\right\rangle \\
\left\langle\sqrt{2} S_{h v} S_{h h}^{*}\right\rangle & \left\langle 2\left|S_{h v}\right|^{2}\right\rangle & \left\langle\sqrt{2} S_{h v} S_{v v}^{*}\right\rangle \\
\left\langle S_{v v} S_{h h}^{*}\right\rangle & \left\langle\sqrt{2} S_{v v} S_{h v}^{*}\right\rangle & \left\langle\left|S_{v v}\right|^{2}\right\rangle
\end{array}\right] .
\end{aligned}
$$

Here $L$ is the nominal number of looks used for averaging, $(\cdot)^{H}$ and $(\cdot)^{*}$ means the Hermitian transposition operator and complex conjugation, respectively, and $\langle\cdot\rangle$ denotes spatial sample averaging. Hence, after multilooking, each pixel in the image is a realization of the $d \times d$ stochastic matrix variable denoted $\mathbf{C}$, and the image is referred to as the MLC covariance image. The dimension $d$ is either 1,2 or 3 , depending on the scattering vector used.

It is commonly assumed that the scattering vector elements $\boldsymbol{\Omega}$ jointly follow a circular complex and multivariate Gaussian distribution [37], denoted as $\boldsymbol{\Omega} \sim \mathcal{N}_{d}^{\mathbb{C}}(\mathbf{0}, \boldsymbol{\Sigma})$, with zero mean vector, true covariance matrix $\boldsymbol{\Sigma}=\mathrm{E}\left\{\boldsymbol{\Omega} \boldsymbol{\Omega}^{H}\right\}=\mathrm{E}\{\mathbf{C}\}$, and dimension $d$. It follows from the Gaussian assumption that if $L \geq d$ and the $\left\{\boldsymbol{\Omega}_{\ell}\right\}_{\ell=1}^{L}$ are independent, then the unnormalized sample covariance matrix, defined as $\mathbf{W}=L \mathbf{C}$, follows the non-singular complex Wishart distribution [38], [39], denoted $\mathcal{W}_{d}^{\mathbb{C}}(L, \boldsymbol{\Sigma})$. The probability density function (pdf) of $\mathbf{W}$ is given as

$$
p_{\mathbf{W}}(\mathbf{W})=\frac{|\mathbf{W}|^{L-d}}{\Gamma_{d}(L)|\boldsymbol{\Sigma}|^{L}} \exp \left(-\operatorname{tr}\left(\boldsymbol{\Sigma}^{-1} \mathbf{W}\right)\right),
$$


where $\operatorname{tr}(\cdot)$ and $|\cdot|$ denote the trace and determinant operators, respectively, and

$$
\Gamma_{d}(L)=\pi^{\frac{d(d-1)}{2}} \prod_{i=1}^{d} \Gamma(L-i+1)
$$

is the multivariate gamma function of the complex kind [40], while $\Gamma(\cdot)$ is the Euler gamma function. Due to normalization by $L$, the sample covariance matrix $\mathbf{C}$ follows a scaled complex Wishart distribution [40], denoted $s \mathcal{W}_{d}^{\mathbb{C}}(L, \boldsymbol{\Sigma})$, whose pdf is

$$
p_{\mathbf{C}}(\mathbf{C})=\frac{L^{L d}|\mathbf{C}|^{L-d}}{\Gamma_{d}(L)|\mathbf{\Sigma}|^{L}} \exp \left(-L \operatorname{tr}\left(\boldsymbol{\Sigma}^{-1} \mathbf{C}\right)\right) .
$$

It should be mentioned that the distributions of both the unnormalized sample covariance matrix $\mathbf{W}$ and the sample covariance matrix $\mathbf{C}$ are presented in (3) and (5), respectively, since the LRT statistic developed in [14] is based on $\mathbf{W}$ and the test statistic proposed in the following is based on C. For single-channel data, i.e., $d=1$, the multilook covariance matrix becomes a scalar intensity and (5) reduces to the gamma distribution.

\section{Proposed Polarimetric Change detector}

Let $\mathcal{A}=\{\mathbf{A}(i, j) ; 1 \leq i \leq I, 1 \leq j \leq J\}$ and $\mathcal{B}=\{\mathbf{B}(i, j) ; 1 \leq i \leq I, 1 \leq j \leq J\}$ be two equal-sized and co-registered MLC PolSAR images acquired over the same geographical area at times $t_{a}$ and $t_{b}$, where $I$ and $J$ are the number of rows and columns of the images, respectively. It is assumed that $\mathcal{A}$ and $\mathcal{B}$ are acquired with the same original resolution, that they are geometrically corrected, and radiometrically calibrated.

To determine whether a change has occured at position $(i, j)$ in the image during the time interval $\left[t_{a}, t_{b}\right]$, we need to compare the PolSAR measurements $\mathbf{A}(i, j)$ and $\mathbf{B}(i, j)$. These are assumed to be statistically independent sample covariance matrices with dimension $d \times d$, where $d$ is the number of polarimetric channels, defined on the cone of Hermitian and positive definite matrices. We further assume that both $\mathbf{A}$ and B both follow scaled complex Wishart distributions, possibly with different distribution parameters, which is denoted

$$
\mathbf{A} \sim \operatorname{s\mathcal {W}}_{d}^{\mathbb{C}}\left(L_{a}, \boldsymbol{\Sigma}_{a}\right) \text { and } \mathbf{B} \sim s \mathcal{W}_{d}^{\mathbb{C}}\left(L_{b}, \boldsymbol{\Sigma}_{b}\right) .
$$

\section{A. ENL Estimation}

The aim is to perform a fully automatic computation of the test statistic for unsupervised change detection in multitemporal multilook PolSAR images. We therefore want an automatic estimate of the ENL, and have applied the method proposed in [35] to avoid manual selection of regions of interest. This method is based on the maximum likelihood estimator for the ENL under the complex Wishart model. The ENL estimate is extracted from an empirical density of small sample estimates calculated in a sliding window which traverses the whole image. The overall distribution of estimates is expected to be dominated by estimates computed from homogeneous samples. Therefore, the mode value of the density maximum can be used as an estimate of the ENL

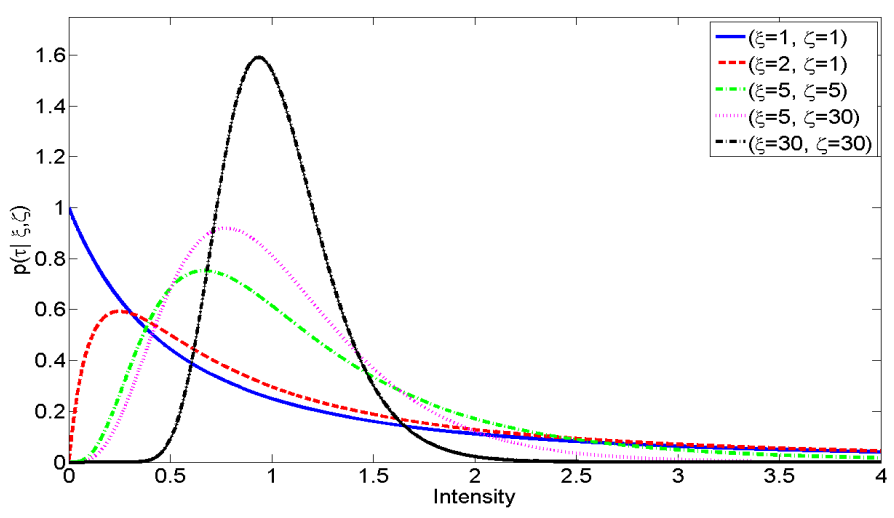

Fig. 1. Flexibility of FS distribution $\mathcal{F S}$ of the unit mean with different pairs of shape parameters $(\xi, \zeta)$.

[35]. Note that underestimation of the ENL may occur due to the presence of texture or a lack of homogeneous areas in the image. It is worth to mention that the formulation of the sample covariance matrix in (2) is not valid for spatially correlated samples and should be divided by the ENL and not by the nominal number of looks. Hence, (2) should be scaled by the ENL to achieve the true covariance matrix. Nevertheless, this is irrelevant for this study because the scale factor is cancelled out by the inverse product in the test statistic introduced in Section III-B.

\section{B. The Complex Hotelling-Lawley Trace Statistic}

The complex-kind Hotelling-Lawley trace statistic is defined as [31]

$$
\tau_{\mathrm{HLT}}=\operatorname{tr}\left(\mathbf{A}^{-1} \mathbf{B}\right) .
$$

In case of equality of covariance matrices $\mathbf{A}$ and $\mathbf{B}$, the value of the test statistic is equal to the polarimetric dimension, i.e., $\tau_{\text {HLT }}=d$. The operator $\tau_{\text {HLT }}$ compacts the matrix-variate quotient into a scalar measure, that can be hypothesis tested. We use the HLT statistic to measure the similarity between the two covariance matrices $\mathbf{A}$ and $\mathbf{B}$, and perform change detection by choosing between the hypotheses:

$$
\left\{\begin{array}{l}
H_{0}: \boldsymbol{\Sigma}_{a}=\boldsymbol{\Sigma}_{b} \\
H_{1}: \boldsymbol{\Sigma}_{a} \neq \mathbf{\Sigma}_{b}
\end{array}\right.
$$

The null hypothesis $\left(H_{0}\right)$ corresponds to no-change and the alternative hypothesis $\left(H_{1}\right)$ to change. In case of singlechannel data, the test statistic reduces to a standard ratio operator between multilook intensities [5]. A threshold selection process can be applied to the test statistic $\tau_{\text {HLT }}$ to distinguish quantitatively between $H_{0}$ and $H_{1}$ (see Section III-D). The pdfs of $\mathbf{A}$ and $\mathbf{B}$ may differ due to different values not only of the covariance matrices but also of the ENLs. The hypothesis tests in this paper are developed with distinct $L_{a} \neq L_{b}$ for generality, and these can be estimated from the separate images. Nevertheless, in the experiments the ENL values are set to be equal, assuming that both images are subject to the same multilook processing. 


\section{Sampling Distribution of the HLT Statistic}

It is difficult to derive an exact sampling distribution for the HLT statistic under the assumption of complex Wishart distributed matrices. As noted in [33] and [34], a scaled version of the real-kind HLT statistic will follow the Fdistribution in the case of $d=1$. We thus assume that the FS distribution [41], which is an F-distribution extended with a location parameter, is an acceptable model also for the matrix-kind HLT with $d=2$ or 3 . It is therefore used as an approximation to the true distribution, denoted

$$
\tau_{\mathrm{HLT}} \sim \mathcal{F} \mathcal{S}(\xi, \zeta, \mu)
$$

where $\mu=\mathrm{E}\left\{\tau_{\mathrm{HLT}}\right\}>0$ is a location parameter, and $\xi>0$ and $\zeta>0$ are two shape parameters. The FS distribution is given by [41]

$$
p_{\tau}(t)=\frac{\Gamma(\xi+\zeta)}{\Gamma(\xi) \Gamma(\zeta)} \frac{\xi}{\mu(\zeta-1)} \frac{\left(\frac{\xi}{\mu(\zeta-1)} t\right)^{\xi-1}}{\left(\frac{\xi}{\mu(\zeta-1)} t+1\right)^{\xi+\zeta}}
$$

In the above formulation, we remove the subscript of $\tau_{\mathrm{HLT}}$ for simplicity. Note that $t$ is a realization of $\tau$.The parametrization used here is slightly different from the one used in [42], in insisting that $\mu$ should be identical to the actual mean [41]. The FS distribution tends to the gamma distribution when $\zeta \rightarrow \infty$ and to the inverse gamma distribution when $\xi \rightarrow \infty$ [43]. The flexibility of the FS distribution is controlled with the shape parameters to steer between heavy-headed and heavy-tailed distributions [43], as shown in Fig. 1. The FS pdf is obtained as the Mellin convolution of a gamma pdf by an inverse gamma pdf [41]. The $\nu^{\text {th }}$ order moments can be expressed in terms of the distribution parameters as [42]

$$
m_{\nu}^{(\mathrm{FS})}\{\tau\}=\left(\frac{(\zeta-1) \mu}{\xi}\right)^{\nu} \frac{\Gamma(\xi+\nu)}{\Gamma(\xi)} \frac{\Gamma(\zeta-\nu)}{\Gamma(\zeta)} .
$$

From (11), we extract the first, second, and third-order moments of the FS distribution as

$$
\begin{gathered}
m_{1}^{(\mathrm{FS})}=\mu \\
m_{2}^{(\mathrm{FS})}=\frac{\xi+1}{\xi} \frac{\zeta-1}{\zeta-2} \mu^{2} \\
m_{3}^{(\mathrm{FS})}=\frac{(\xi+1)(\xi+2)}{\xi^{2}} \frac{(\zeta-1)^{2}}{(\zeta-2)(\zeta-3)} \mu^{3} .
\end{gathered}
$$

The remaining problem is to determine the parameters of the FS distribution. The method proposed in this study is to match the population moments of the FS distribution from (11) with the population moments of the HLT statistic.

We here present expressions for the moments of the HLT statistic that have been derived using results from [44] under the null hypothesis. The mean of $\tau_{\text {HLT }}$ is given as

$$
m_{1}^{(\mathrm{HLT})}=\mathrm{E}\left\{\tau_{\mathrm{HLT}}\right\}=\frac{d L_{a}}{Q_{a}} .
$$

where $Q_{a}=L_{a}-d$. The second-order moment is

$$
\begin{aligned}
m_{2}^{(\mathrm{HLT})} & =\mathrm{E}\left\{\tau_{\mathrm{HLT}}^{2}\right\} \\
& =\frac{L_{a}^{2}}{Q_{a}^{3}-Q_{a}}\left(d^{2}\left(Q_{a}+\frac{1}{L_{b}}\right)+d\left(\frac{Q_{a}}{L_{a}}+1\right)\right) .
\end{aligned}
$$

and the third-order moment is given as

$$
\begin{aligned}
& m_{3}^{(\mathrm{HLT})}=\mathrm{E}\left\{\tau_{\mathrm{HLT}}^{3}\right\} \\
& =\frac{L_{a}^{3}}{Q_{a}^{5}-5 Q_{a}^{3}+4 Q_{a}} \times\left[d^{3}\left(\left(Q_{a}^{2}-1\right)+\frac{3 Q_{a}}{L_{b}}+\frac{4}{L_{b}^{2}}\right)\right. \\
& +d^{2}\left(3 Q_{a}+\frac{3\left(Q_{a}^{2}+2\right)}{L_{b}}+\frac{6 Q_{a}}{L_{b}^{2}}\right) \\
& \left.+d\left(4+\frac{6 Q_{a}}{L_{b}}+\frac{2 Q_{a}^{2}}{L_{b}^{2}}\right)\right] .
\end{aligned}
$$

As seen in the expressions, the first, second, and third-order moments of $\tau_{\mathrm{HLT}}$ are all functions of the number of looks, $L_{a}$ and $L_{b}$, and the dimension of the PolSAR data, $d$. They are all independent of the scale matrix $\Sigma$, which cancels out under the null hypothesis, when it is assumed equal in both images. It should also be noticed from (16) and (17) that the HLT moments go to infinity when $Q_{a}=0,1,2$. Therefore, it is pertinent to multilook such that $Q_{a}>2$ or $L_{a}>d+2$. In the experiments we make the realistic assumption that the PolSAR image pair has been subject to the same multilook processing, which leads to some simplification with $L_{a}=L_{b}=L$ in (16) and (17).

In the more general case, we seek expressions for the parameters of the FS distribution in terms of the distribution parameters of the scaled Wishart matrices $\mathbf{A}$ and B, i.e., $L_{a}$, $L_{b}$, and $d$. The solution for $\mu, \xi$, and $\zeta$ are defined by the equation system

$$
m_{\nu}^{(\mathrm{FS})}(\xi, \zeta, \mu)=m_{\nu}^{(\mathrm{HLT})}\left(L_{a}, L_{b}, d\right), \quad \nu=1,2,3 .
$$

The location parameter $\mu$ of the FS distribution is computed analytically from (15). To match the second and third-order moments and retrieve the shape parameters $\xi$ and $\zeta$, we use minimum distance optimization [45] to solve

$$
\begin{gathered}
\epsilon^{2}=\sum_{\nu=2}^{3}\left(m_{\nu}^{(\mathrm{HLT})}-m_{\nu}^{(\mathrm{FS})}\right)^{2}, \\
(\hat{\xi}, \hat{\zeta})=\arg \left\{\min _{(\xi, \zeta)}\left\{\epsilon^{2}\right\}\right\} .
\end{gathered}
$$

In this numerical problem, the values of $L_{a}$ and $L_{d}$ are assumed known and need to be replaced with ENLs estimated from the input PolSAR images. Estimation of the ENLs is a critical point in this procedure. To do this accurately and automatically, we use the unsupervised method described in Section III-A.

In conclusion, the fitting of the distribution parameters, $(\xi, \zeta, \mu)$, depends on the data only through the estimates of $L_{a}$ and $L_{b}$, based on an analysis of each entire image, and not through samples of $\tau_{\mathrm{HLT}}$. 


\section{CFAR-Type Thresholding}

Let $p_{\tau}(t)$ denote the sampling distribution of $\tau_{\mathrm{HLT}}$ under $H_{0}$, estimated as described in Section III-C. In the CFARtype $^{1}$ approach we set a desired false alarm probability $P_{\mathrm{fa}}$, often expressed in percent as the significance level $\alpha_{c}=$ $100 \cdot P_{\mathrm{fa}}$, and determine the threshold from the sampling distribution. The adopted decision rule depends on the type of change we want to detect. Changes from $\mathbf{A}$ to $\mathbf{B}$ can either cause an increase in $\tau_{\mathrm{HLT}}=\operatorname{tr}\left(\mathbf{A}^{-1} \mathbf{B}\right)$ or decrease in $\tau_{\text {HLT }}=\operatorname{tr}\left(\mathbf{A}^{-1} \mathbf{B}\right)$. Alternatively, we may use the HLT statistic reversely, as $\tau_{\mathrm{HLT}}^{\prime}=\operatorname{tr}\left(\mathbf{B}^{-1} \mathbf{A}\right)$ to characterize the change direction. Generally, the type of change and its effect on $\tau_{\text {HLT }}$ are not known prior to detection and both tails must be thresholded. This gives the two-sided test as

$$
T_{\mathrm{lo}} \underset{H_{0}}{\stackrel{H_{1}}{\gtrless}} \tau_{\text {HLT }} \underset{H_{0}}{\stackrel{H_{1}}{\gtrless}} T_{\text {up }} \text {. }
$$

where the inequality truth denotes the associated hypothesis decision, $H_{0}$ for no-change and $H_{1}$ for change, $T_{\text {up }}$ defines the upper $\alpha_{c}$ percentile of $p_{\tau}(t)$ corresponding to high values of $\tau_{\mathrm{HLT}}$, and $T_{\mathrm{lo}}$ defines the lower $\alpha_{c}$ percentile of $p_{\tau}(t)$ corresponding to low values of $\tau_{\text {HLT }}$. Then, for a desired false alarm probability $P_{\text {fa }}$, the thresholds $T_{\text {up }}$ and $T_{\text {lo }}$ can be determined from

$$
P_{\mathrm{fa}}=\int_{0}^{T_{\mathrm{lo}}} p_{\tau}\left(t \mid H_{0}\right) d t+\int_{T_{\text {up }}}^{\infty} p_{\tau}\left(t \mid H_{0}\right) d t .
$$

With two thresholds in (21), the definition in (22) is adjusted such that the two rejection regions together specify the desired false alarm probability of $P_{\mathrm{fa}}$.

We remark that a change manifested as high or low $\tau_{\text {HLT }}$ values cannot be interpreted unequivocally as backscatter increase or decrease in the multipolarimetric case. The test statistic captures changes in both polarimetry and intensity, where we interpret the former as changes in the matrix structure and in the balance between the polarimetric channels, and the latter to changes in an overall intensity averaged over the channels. An increase or decrease in the test statistic can thus be caused by various circumstances, and we cannot provide any general interpretation of the projection it exerts on the data. Eigendecomposition theory can be used to analyze polarimetric changes in terms of scattering mechanisms, as proposed by Alonso-González et al. in [46], [47].

We may also calculate both $\operatorname{tr}\left(\mathbf{A}^{-1} \mathbf{B}\right)$ and $\operatorname{tr}\left(\mathbf{B}^{-1} \mathbf{A}\right)$ and take the maximum value. The combined test is given as

$$
\max \left[\tau_{\mathrm{HLT}}, \tau_{\mathrm{HLT}}^{\prime}\right] \underset{H_{0}}{\stackrel{H_{1}}{\gtrless}} T_{\text {up }},
$$

which maps all types of change, from both $\tau_{\mathrm{HLT}}$ and $\tau_{\mathrm{HLT}}^{\prime}$, into high values. We refer to this as the maximum HLT statistic. The combined threshold $T_{\text {up }}$ can be derived from

$$
P_{\mathrm{fa}}=2 \int_{T_{\text {up }}}^{\infty} p_{\tau}\left(t \mid H_{0}\right) d t
$$

\footnotetext{
${ }^{1}$ The HLT test is not CFAR, but we use the CFAR principle to determine the threshold.
}

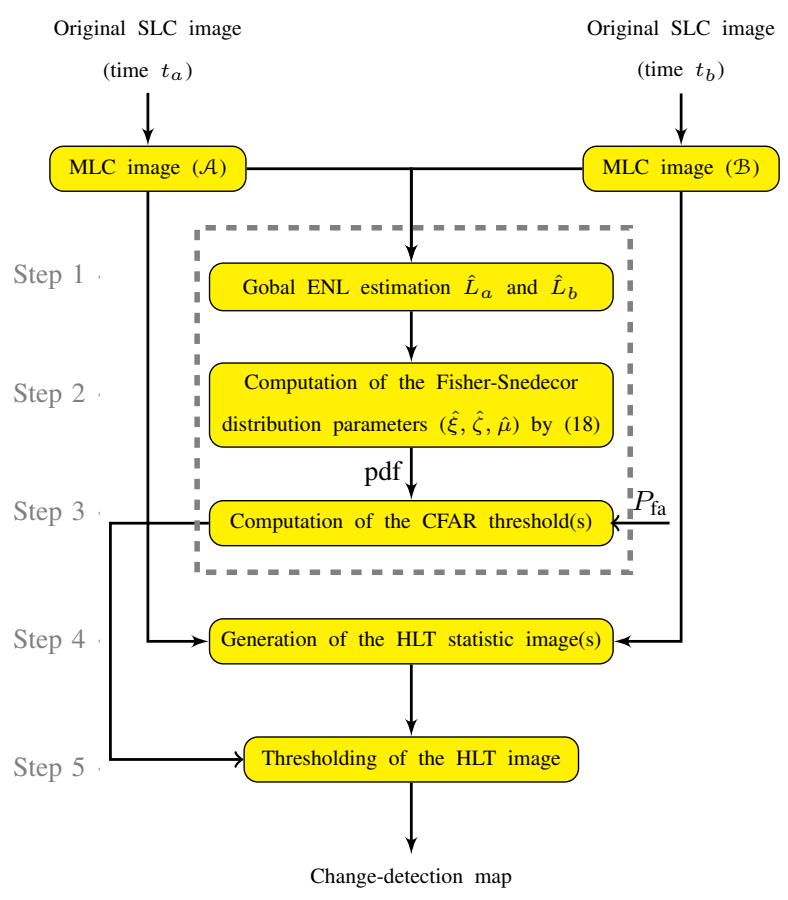

Fig. 2. General block diagram of the proposed change detection algorithm for PolSAR data. The dashed box represents the pre-processing stages, before comparing the two images.

since both the lower and upper tails in (22) are now mapped to the same tail.

By specifying $P_{\mathrm{fa}}$ and then solving (24) for the required threshold, a CFAR-type change detector is obtained. In the two-sided test in (22), we could generalize to use different percentiles on the two tails to introduce different risks associated with the types of change. Other methods used different criteria to determine the threshold. One alternative is the K\&I method [28], which was elaborated and adopted to SAR images in [2] and [3]. It minimizes the total classification error under simplified assumptions, including both false alarms and missed detections. In this paper, we confine ourselves to the CFARtype algorithm, in order to perform a fair comparison between the proposed method and the LRT detector, since we are more interested in the quality of the test statistic than the thresholding method.

\section{E. Summary}

In summary, the general block diagram of the proposed unsupervised change detection approach in multipolarization SAR data is shown in Fig. 2, which is made up of five main steps:

1) Separate ENL estimation for each image. If the images are produced with the same acquisition mode and processing parameters, the estimates are averaged;

2) Computation of the FS distribution parameters from $d$, $L_{a}$, and $L_{b}$;

3) Computation of the CFAR threshold(s) for the desired $P_{\mathrm{fa}}$;

4) Generation of the HLT test image(s) from two MLC images;

5) Application of the decision criterion. 


\section{Likelihood Ratio Test Statistic}

An LRT for equality of two complex Wishart matrices was first proposed by Conradsen et al. [14]. For the two unnormalized sample covariance matrices $\mathbf{M}=L_{a} \mathbf{A}$ and $\mathbf{N}=L_{b} \mathbf{B}$ that both follow the complex Wishart distribution, i.e., $\mathbf{M} \sim \mathcal{W}_{d}^{\mathbb{C}}\left(L_{a}, \boldsymbol{\Sigma}_{a}\right)$ and $\mathbf{N} \sim \mathcal{W}_{d}^{\mathbb{C}}\left(L_{b}, \boldsymbol{\Sigma}_{b}\right)$, we may examine if $\mathbf{M}$ equals $\mathbf{N}$ by considering the null hypothesis against the alternative hypothesis in (8). The Wishart LRT statistic was derived as [14]

$$
Q=\frac{\left(L_{a}+L_{b}\right)^{d\left(L_{a}+L_{b}\right)}}{L_{a}^{d L_{a}} L_{b}^{d L_{b}}} \frac{|\mathbf{M}|^{L_{a}}|\mathbf{N}|^{L_{b}}}{|\mathbf{M}+\mathbf{N}|^{L_{a}+L_{b}}} .
$$

When the values of $L_{a}$ and $L_{b}$ are assumed equal, and replaced with an average of the estimated ENLs, i.e., $L=\left(\hat{L}_{a}+\hat{L}_{b}\right) / 2$, we get

$$
\ln Q=L(2 d \ln 2+\ln |\mathbf{M}|+\ln |\mathbf{N}|-2 \ln |\mathbf{M}+\mathbf{N}| .
$$

The test statistic for change detection based on the LRT is given as:

$$
\tau_{\mathrm{LRT}}=-2 \rho \ln Q,
$$

where

$$
\rho=1-\frac{2 d^{2}-1}{4 L d} .
$$

The sampling distribution of $\tau_{\mathrm{LRT}}$ is approximated by [14]

$$
\tau_{\mathrm{LRT}} \sim \chi^{2}\left(d^{2}\right)+\omega_{2}\left[\chi^{2}\left(d^{2}+4\right)-\chi^{2}\left(d^{2}\right)\right] .
$$

where $\chi^{2}(\vartheta)$ denotes a central $\chi^{2}$ distribution with $\vartheta$ degrees of freedom and

$$
\omega_{2}=-\frac{d^{2}}{4}\left(1-\frac{1}{\rho}\right)^{2}+\frac{7 d^{2}\left(d^{2}-1\right)}{96 L^{2} \rho^{2}} .
$$

A test with a desired false alarm probability $P_{\mathrm{fa}}$ is given as

$$
\tau_{\text {LRT }} \underset{H_{0}}{\stackrel{H_{1}}{\gtrless}} T_{\text {up }} \text {. }
$$

The threshold $T_{\text {up }}$ is the upper $\alpha_{c}$ percentile of the asymptotic distribution in (29).

In the one-dimensional case, $Q$ reduces to a generalized LRT statistic for equality of two gamma distributed intensities. The test statistic maps all change pixels to the upper tail of the histogram. This means that the LRT is intrinsically a onesided test, whereas the HLT requires the combined test in (23) to obtain the same test (change versus no-change).

\section{EXPERIMENTAL RESUlts AND ANALYSis}

To evaluate the capability of the proposed detector, the LRT has been implemented for comparison. In the following, these two detection methods are tested with both simulated and real PolSAR images. The test data sets include pairs of simulated quadruple polarisation (quad-pol) images, and RADARSAT-2 quad-pol scenes over an urban area in Suzhou, East China. It is noted that both methods can be applied to both single and multichannel SAR data, but here they are applied only to multichannel data.

\section{A. Experiment I: Simulated Multilook PolSAR Data}

1) The model fit of $\tau_{H L T}$ for the no-change class: In the first experiment, we generate two multiclass matrix images that follow a scaled complex Wishart distribution with $L=12$ and class-specific $\boldsymbol{\Sigma}$ matrices. We now evaluate the performance of the methods on quad-pol data. The simulated PolSAR images are $250 \times 250$ pixels in size and have three polarimetric channels. To be realistic, the distribution of each class is parameterized by a scale matrix $\boldsymbol{\Sigma}_{\mathrm{j}}$ estimated by averaging homogeneous regions in a real data set. The scale matrix of class $\mathrm{j}$ is defined as [48]

$$
\boldsymbol{\Sigma}_{\mathrm{j}}=\mu_{\mathrm{j}} \boldsymbol{\Gamma}_{\mathrm{j}}
$$

where $\Gamma_{\mathrm{j}}$ is the internal covariance structure matrix carrying polarimetric information, normalized such that the determinant $\left|\boldsymbol{\Gamma}_{\mathrm{j}}\right|=1$; and $\mu_{\mathrm{j}}=\left|\boldsymbol{\Sigma}_{\mathrm{j}}\right|^{1 / d}$ is the geometric mean intensity (GMI) of each class [48]. The GMI is a measure of the combined intensity in the polarization channels. $\mu_{\mathrm{j}}$ is similar to the span, but is equivalent to the geometric mean of the eigenvalues rather than the sum. Fig. 3(a) shows the Pauli composite image $(\mathrm{R}=\mathrm{HH}-\mathrm{VV}, \mathrm{G}=\mathrm{HV}, \mathrm{B}=\mathrm{HH}+\mathrm{VV})$ of the simulated test pattern at time $t_{a}$. Class 3 is simulated with the polarimetric properties of water. Class 7 was simulated with the polarimetric properties of an urban area. The other classes are simulated with the properties of agricultural crops and vegetation areas. Another instance of the PolSAR image (not shown) with the same statistical properties was realized to represent time $t_{b}$, assuming that no change has occurred during the time interval $\left[t_{a}, t_{b}\right]$. The pair is used to assess the model fit of $\tau_{\text {HLT }}$ under the $H_{0}$ assumption (no-change scenario). A Monte Carlo simulation is used to synthesize the PolSAR data sets with 100 repetitions for each pair.

We derive the FS distribution parameters as in Section III-C and generate $\tau_{\text {HLT }}$ based on the image pair. The images are simulated with no spatial correlation, such that the ENL is equal to the nominal number of looks. To assess whether the FS distribution is a good approximation to the HLT data, we apply the Kolmogorov-Smirnov (KS) and Anderson-Darling (AD) goodness-of-fit (GoF) hypothesis tests [49]. The KS statistic for a given cumulative distribution function (cdf) $F(x)$ is

$$
D_{n}=\sup _{x}\left|F_{n}(x)-F(x)\right|,
$$

where $\sup _{x}$ is the supremum of the set of distances, $F_{n}$ is the empirical distribution function (edf), $n$ is the number of observations, and $D_{n}$ is a measure of the deviation of empirical cdf from an hypothesized cdf. The AD statistic $\left(A^{2}\right)$ is defined as

$$
A^{2}=-n-\sum_{i=1}^{n} \frac{2 i-1}{n}\left[\ln F\left(x_{i}\right)+\ln \left(1-F\left(x_{n-i+1}\right)\right)\right] .
$$

The GoF tests are used to test the accuracy of the FS model 


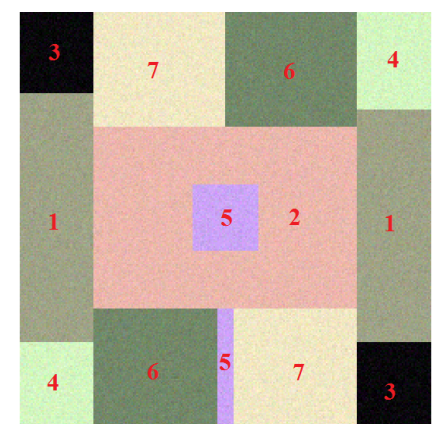

(a)

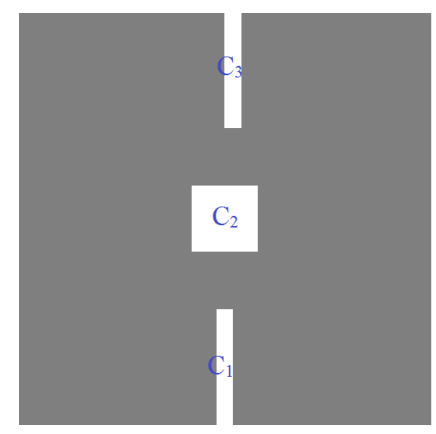

(c)

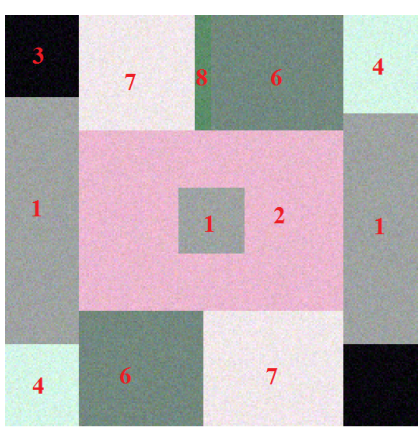

(b)

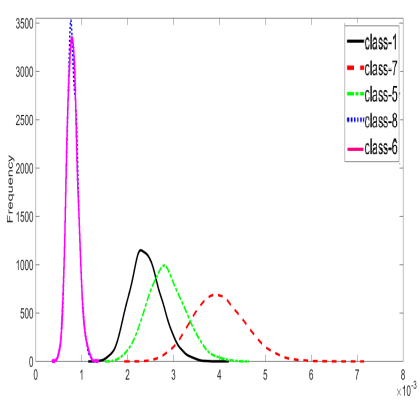

(d) pdf of $\operatorname{tr}\left(\boldsymbol{\Gamma}_{\mathbf{j}}^{-1} \mathbf{A}\right)$
Fig. 3. Experiment with a pair of simulated 12-look quad-pol scaled Wishart distributed images. (a) and (b) Pauli decomposition composite images with class labels at two different times with changes. (c) theoretical result of change detection, where white is change and gray is no-change. (d) class histograms of $\operatorname{tr}\left(\boldsymbol{\Gamma}_{\mathrm{j}}^{-1} \mathbf{A}\right)$ for the change classes (classes 1, 5, 6, 7 and 8 ) in the simulated test patterns. The change areas $C_{1}$ to $C_{3}$ are discussed in the main text and summarized in Table II.

approximation as the sampling distribution for the hypothesis ${ }^{2}$ :

$$
\left\{\begin{array}{l}
\mathscr{H}_{0}: \text { The HLT data follows the FS model, } \\
\mathscr{H}_{1}: \text { The HLT data does not follow the FS model. }
\end{array}\right.
$$

We produce a $\tau_{\text {HLT }}$ image from the $H_{0}$ image pair and plug it into the GoF tests to obtain the associated $p$-value. An exact sampling distribution would produce an average $p$-value of 0.5 . Deviations from this quantify the imperfection of the approximation. The decision to reject the null hypothesis is based on comparing the $p$-values with the specified probability of false alarm $P_{\mathrm{fa}}$ in the test

$$
p \text {-value } \underset{\mathscr{H}_{1}}{\stackrel{\mathscr{H}_{0}}{\gtrless}} P_{\text {fa }} \text {. }
$$

Table I presents the average values of $D_{n}, A^{2}$, and $p$-values with corresponding variances. If the tests reject $\mathscr{H}_{0}$ at $P_{\mathrm{fa}}=$ 0.05 , the fitted model would be rejected. It is evident from the $p$-values that we have no strong evidence against $\mathscr{H}_{0}$ in all cases (different number of looks and polarimetric dimensions) from our quantitative analysis. This indicates that the fitted FS distribution can satisfactorily model the no-change class.

${ }^{2}$ Note that the different notation is used here for the GoF hypotheses to avoid confusion with the pixel-wise change detection hypotheses in Section III-D.
TABLE I

AVERAGE $D_{n}, A^{2}$, AND $p$-VALUES WITH CORRESPONDING VARIANCES

\begin{tabular}{|c|c|c|c|c|c|c|c|c|}
\hline \multicolumn{9}{|c|}{ Quad-pol } \\
\hline & \multicolumn{4}{|c|}{$\mathrm{KS}$ test } & \multicolumn{4}{|c|}{ AD test } \\
\hline Looks & $D_{n}$ & $\sigma_{D_{n}}^{2}$ & $p$ & $\sigma_{p}^{2}$ & $A^{2}$ & $\sigma_{A^{2}}^{2}$ & $p$ & $\sigma_{p}^{2}$ \\
\hline 7 & 0.049 & $4 \mathrm{e}-7$ & 0.65 & 0.12 & 1.50 & $3 e-4$ & 0.59 & 0.08 \\
\hline 8 & 0.035 & $5 e-4$ & 0.59 & 0.09 & 2.40 & $5 e-4$ & 0.65 & 0.07 \\
\hline 9 & 0.055 & $2 \mathrm{e}-3$ & 0.54 & 0.07 & 2.25 & $5 e-6$ & 0.57 & 0.09 \\
\hline 16 & 0.031 & $3 e-5$ & 0.58 & 0.11 & 1.32 & $4 e-8$ & 0.42 & 0.12 \\
\hline 25 & 0.039 & $8 \mathrm{e}-2$ & 0.56 & 0.08 & 1.22 & $6 e-4$ & 0.61 & 0.14 \\
\hline 36 & 0.045 & $2 \mathrm{e}-1$ & 0.54 & 0.06 & 1.56 & $4 e-5$ & 0.51 & 0.18 \\
\hline 49 & 0.102 & $1 \mathrm{e}-5$ & 0.47 & 0.08 & 2.51 & $7 e-3$ & 0.60 & 0.12 \\
\hline 64 & 0.108 & $5 e-4$ & 0.65 & 0.13 & 2.74 & $9 e-2$ & 0.57 & 0.08 \\
\hline 81 & 0.104 & $8 \mathrm{e}-3$ & 0.53 & 0.11 & 1.83 & $1 \mathrm{e}-1$ & 0.60 & 0.13 \\
\hline 100 & 0.095 & $2 \mathrm{e}-6$ & 0.46 & 0.05 & 2.11 & $2 \mathrm{e}-9$ & 0.65 & 0.16 \\
\hline \multicolumn{9}{|c|}{ Dual-pol } \\
\hline Looks & $D_{n}$ & $\sigma_{D_{n}}^{2}$ & $p$ & $\sigma_{p}^{2}$ & $A^{2}$ & $\sigma_{A^{2}}^{2}$ & $p$ & $\sigma_{p}^{2}$ \\
\hline 7 & 0.017 & $4 e-3$ & 0.64 & 0.17 & 2.50 & $5 e-6$ & 0.65 & 0.11 \\
\hline 8 & 0.015 & $5 e-4$ & 0.62 & 0.15 & 1.50 & $7 e-2$ & 0.57 & 0.05 \\
\hline 9 & 0.085 & $6 e-7$ & 0.57 & 0.13 & 1.83 & $1 \mathrm{e}-6$ & 0.53 & 0.08 \\
\hline 16 & 0.092 & $4 e-5$ & 0.59 & 0.08 & 3.04 & $2 \mathrm{e}-5$ & 0.55 & 0.12 \\
\hline 25 & 0.152 & $1 \mathrm{e}-3$ & 0.57 & 0.06 & 1.99 & $8 \mathrm{e}-4$ & 0.60 & 0.13 \\
\hline 36 & 0.096 & $2 \mathrm{e}-8$ & 0.47 & 0.07 & 2.04 & $7 e-8$ & 0.51 & 0.12 \\
\hline 49 & 0.086 & $9 e-9$ & 0.51 & 0.04 & 1.58 & $9 e-6$ & 0.48 & 0.11 \\
\hline 64 & 0.103 & $5 e-5$ & 0.53 & 0.13 & 2.31 & $5 e-4$ & 0.53 & 0.05 \\
\hline 81 & 0.121 & $8 \mathrm{e}-4$ & 0.47 & 0.05 & 2.46 & $3 e-5$ & 0.52 & 0.04 \\
\hline 100 & 0.054 & $4 \mathrm{e}-7$ & 0.51 & 0.06 & 2.34 & $4 e-9$ & 0.58 & 0.07 \\
\hline
\end{tabular}
FOR SIMULATED DUAL-POL AND QUAD-POL PAIRS WITH DIFFERENT LEVELS OF MULTILOOKING

TABLE II

THREE CHANGE AREAS AND THEIR CHANGE PARAMETERS WITH RESPECT TO EACH OTHER IN THE SYNTHESIZED IMAGES

\begin{tabular}{|c|c|c|}
\hline Change area & From $\longrightarrow$ To & Change parameters \\
\hline $\mathrm{C}_{1}$ & $5 \longrightarrow 7$ & $\mu_{5}<\mu_{7}, \boldsymbol{\Gamma}_{5} \neq \boldsymbol{\Gamma}_{7}$ \\
\hline $\mathrm{C}_{2}$ & $5 \longrightarrow 1$ & $\mu_{5}>\mu_{1}, \boldsymbol{\Gamma}_{5} \neq \boldsymbol{\Gamma}_{1}$ \\
\hline $\mathrm{C}_{3}$ & $6 \longrightarrow 8$ & $\mu_{6}=\mu_{8}, \boldsymbol{\Gamma}_{6} \neq \boldsymbol{\Gamma}_{8}$ \\
\hline
\end{tabular}

2) The change detection scenario: In the second stage, we synthesize an image with three change areas. First we have imposed GMI and polarimetric changes in the data such that two patches of class 5 in the first image are changed into class 7 and class 1 in the second image. We have also changed a patch of class 6 into a new class 8 to simulate a change only in polarimetric information with the same GMI information (constant GMI, but different matrix structure). Table II summarizes the change areas with the change parameters. The simulated change can mimic both land cover change and seasonal change. A Pauli image with the imposed changes is shown in Fig. 3(b), while Fig. 3(c) shows the reference change map with changed pixels in white and unchanged in gray.

Let $\mathbf{A} \sim s \mathcal{W}_{d}^{\mathbb{C}}\left(L, \boldsymbol{\Sigma}_{\mathrm{j}}\right)$ denote the covariance matrices drawn from class $\mathrm{j}$, which is characterized by (32). The transformation $\operatorname{tr}\left(\boldsymbol{\Gamma}_{\mathbf{j}}^{-1} \mathbf{A}\right)$ is utilized to compact the matrix-variate data into a scalar, such that the variations in GMI between the classes can be visualized. It is easily shown that

$$
\mathrm{E}\left\{\operatorname{tr}\left(\boldsymbol{\Gamma}_{\mathrm{j}}^{-1} \mathbf{A}\right)\right\}=\mu_{\mathrm{j}} \mathrm{E}\left\{\operatorname{tr}\left(\boldsymbol{\Sigma}_{\mathrm{j}}^{-1} \mathbf{A}\right)\right\}=\mu_{\mathrm{j}} d,
$$

and the variance of $\operatorname{tr}\left(\boldsymbol{\Gamma}_{\mathbf{j}}^{-1} \mathbf{A}\right)$ is given as

$$
\operatorname{Var}\left\{\operatorname{tr}\left(\boldsymbol{\Gamma}_{\mathbf{j}}^{-1} \mathbf{A}\right)\right\}=\frac{\mu_{\mathrm{j}}^{2} d}{L} .
$$

Fig. 3(d) shows class histograms of the entity $\operatorname{tr}\left(\boldsymbol{\Gamma}_{j}^{-1} \mathbf{A}\right)$ for 


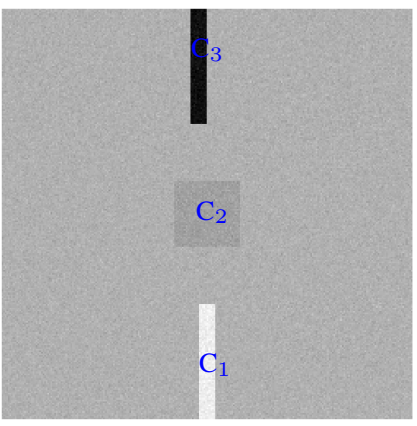

(a) $\log \left(\tau_{\mathrm{HLT}}\right)$

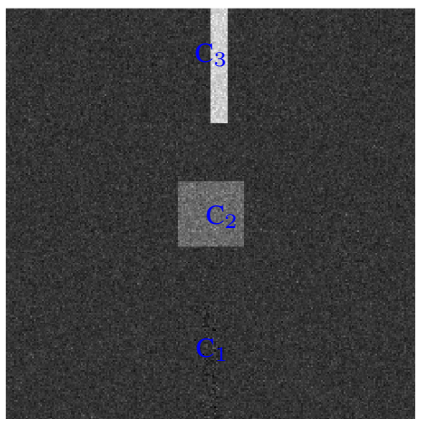

(c) $\log \left(\tau_{\mathrm{HLT}}^{\prime}\right)$

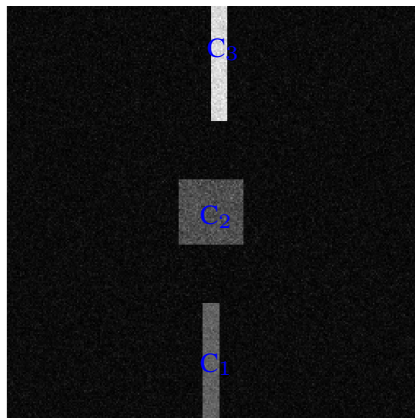

(e) $\log \left(\max \left(\tau_{\mathrm{HLT}}, \tau_{\mathrm{HLT}}^{\prime}\right)\right)$

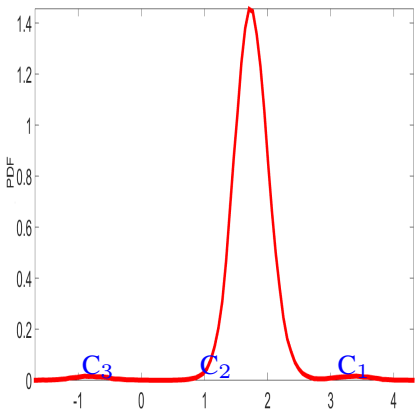

(b) pdf of $\log \left(\tau_{\mathrm{HLT}}\right)$

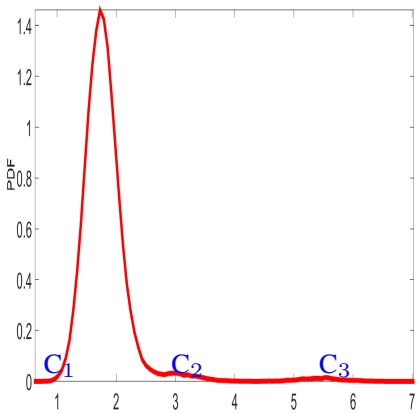

(d) pdf of $\log \left(\tau_{\mathrm{HLT}}^{\prime}\right)$

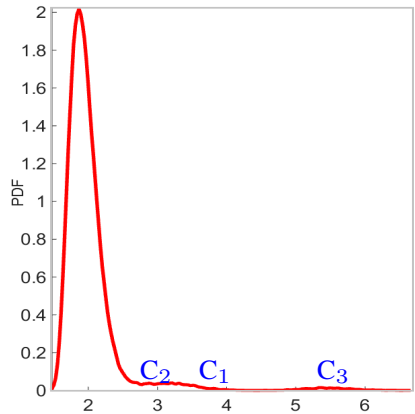

(f) pdf of $\log \left(\max \left(\tau_{\mathrm{HLT}}, \tau_{\mathrm{HLT}}^{\prime}\right)\right)$

Fig. 4. The HLT statistic for Experiment I. (a) logarithm of $\tau_{\text {HLT }}=$ $\operatorname{tr}\left(\mathbf{A}^{-1} \mathbf{B}\right)$. (b) histogram of $\log \left(\tau_{\mathrm{HLT}}\right)$. (c) logarithm of $\tau_{\mathrm{HLT}}^{\prime}=\operatorname{tr}\left(\mathbf{B}^{-1} \mathbf{A}\right)$. (d) histogram of $\log \left(\tau_{\mathrm{HLT}}^{\prime}\right)$. (e) logarithm of $\max \left[\tau_{\mathrm{HLT}}, \tau_{\mathrm{HLT}}^{\prime}\right]$. (f) histogram of $\log \left(\max \left[\tau_{\mathrm{HLT}}, \tau_{\mathrm{HLT}}^{\prime}\right]\right)$. The change areas $\mathrm{C}_{1}$ to $\mathrm{C}_{3}$ are summarized in Table II.

the change classes (classes 1, 5, 6, 7, and 8) in the simulated test patterns. It shows that class 6 and 8 have low GMI values that are very similar. It further shows that the class transitions $5 \rightarrow 1$ and $5 \rightarrow 7$ represent GMI decrease $\left(\mu_{5}>\mu_{1}\right)$ and GMI increase $\left(\mu_{5}<\mu_{7}\right)$. Curves belonging to class 6 and 8 represent the same GMI, i.e., $\mu_{6}=\mu_{8}$; but they have different polarimetric information contained in their covariance structure matrices, i.e., $\boldsymbol{\Gamma}_{6} \neq \boldsymbol{\Gamma}_{8}$.

FS distribution parameters are first derived to obtain the approximate sampling distribution of $\tau_{\mathrm{HLT}}$ under the null hypothesis. The CFAR upper and lower tail thresholds $T_{\mathrm{lo}}$ and $T_{\text {up }}$ are computed at half of the predefined significance level, i.e., $\alpha_{c} / 2$. From the simulated PolSAR images, we generate both operators $\tau_{\mathrm{HLT}}=\operatorname{tr}\left(\mathbf{A}^{-1} \mathbf{B}\right)$ and $\tau_{\mathrm{HLT}}^{\prime}=\operatorname{tr}\left(\mathbf{B}^{-1} \mathbf{A}\right)$. High values of $\tau_{\mathrm{HLT}}$ and $\tau_{\mathrm{HLT}}^{\prime}$ indicate changes in either GMI or

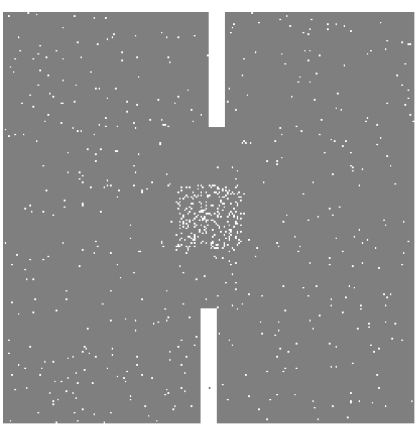

(a) $T_{\mathrm{lo}} \underset{H_{0}}{\stackrel{H_{1}}{\gtrless}} \tau_{\text {HLT }} \underset{H_{0}}{\stackrel{H_{1}}{\gtrless}} T_{\text {up }}$

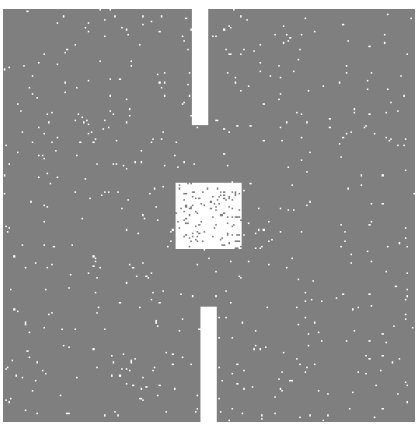

(c) $\max \left(\tau_{\mathrm{HLT}}, \tau_{\mathrm{HLT}}^{\prime}\right) \underset{H_{0}}{\stackrel{H_{1}}{\gtrless}} T_{\text {up }}$

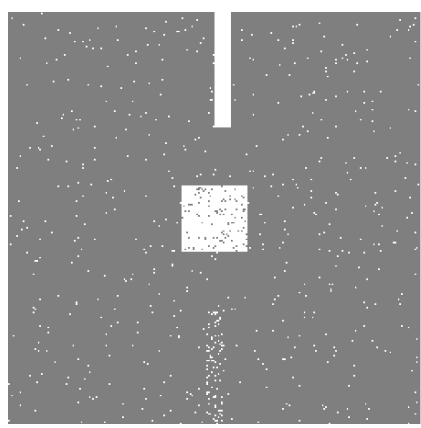

(b) $T_{\text {lo }} \underset{H_{0}}{\stackrel{H_{1}}{\gtrless}} \tau_{\text {HLT }}^{\prime} \underset{H_{0}}{\stackrel{H_{1}}{\gtrless}} T_{\text {up }}$

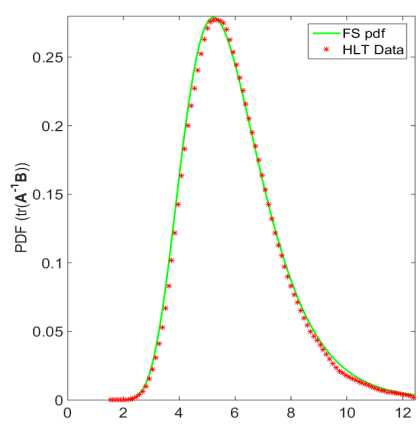

(d) pdf of $\operatorname{tr}\left(\mathbf{A}^{-1} \mathbf{B}\right)$ for the no-change class
Fig. 5. The HLT change detection results for Experiment I. change detection map: rejection of hypothesis of equal covariance matrices at $1 \%$ significance level for (a) two-sided test on $\tau_{\text {HLT }}$ statistic. (b) two sided test on $\tau_{\text {HLT }}^{\prime}$ statistic. (c) combined test. Color legend: white, change; gray, no-change. (d) pdf of the theoretical and observed distribution of $\tau_{\mathrm{HLT}}$ for the no-change class.

polarimetry. Fig. 4(a) is an image of the $\tau_{\mathrm{HLT}}$ statistic on logarithmic scale to enhance the contrast and Fig. 4(b) shows the corresponding histogram. In the histogram, the small peak in the upper tail corresponds to change area $\mathrm{C}_{1}$ with HLT increase in the image. The pixels with HLT decrease in area $\mathrm{C}_{2}$ are absorbed in the main mode and harder to identify. The pixels with a change only in polarimetry in area $\mathrm{C}_{3}$, as the darkest region of the test image, correspond to the small peak in the lower tail. Fig. 4(c) and 4(d) represent an image of the $\tau_{\text {HLT }}^{\prime}$ operator on logarithmic scale and its histogram. In Fig. 4(d), the pixels with $\tau_{\mathrm{HLT}}^{\prime}$ increase constitute the two small peaks in the upper tail (the left peak belongs to the GMI and polarimetric changes in area $\mathrm{C}_{2}$ and the right peak stems from the changes in polarimetry only in area $\mathrm{C}_{3}$ ), whereas the pixels with $\tau_{\text {HLT }}^{\prime}$ decrease in area $\mathrm{C}_{1}$ blend with the main mode. The peaks being lost in the main peak in Fig. 4(b) and 4(d) appear to indicate that the sensitivity is greater in the upper tail of the histograms. This means that both $\tau_{\mathrm{HLT}}$ and $\tau_{\mathrm{HLT}}^{\prime}$ are more sensitive with respect to changes that maps the statistics into the upper tail. Fig. 4(e) and 4(f) illustrate the maximum HLT statistic on logarithmic scale and its histogram with change pixels all mapped to the upper tail.

We then apply the two-sided tests for both $\tau_{\mathrm{HLT}}$ and $\tau_{\mathrm{HLT}}^{\prime}$ as well as the combined test to detect change pixels at the desired significance level $\alpha_{c}$. Fig. 5(a), 5(b), and 5(c) represent the corresponding change results from both two-sided and 


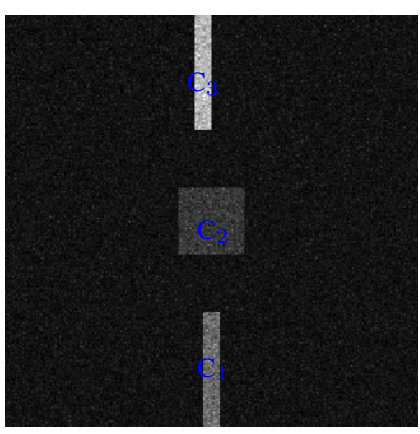

(a) $\tau_{\text {LRT }}$

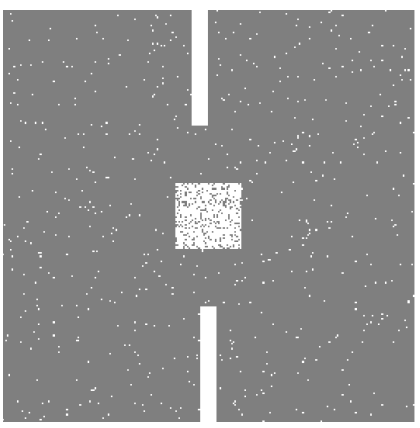

(c) $\tau_{\text {LRT }} \underset{H_{0}}{\stackrel{H_{1}}{\gtrless}} T_{\text {up }}$

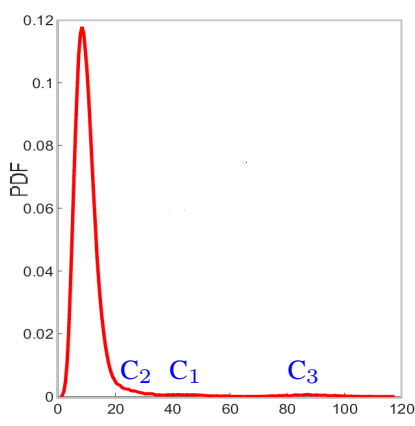

(b) pdf of $\tau_{\text {LRT }}$

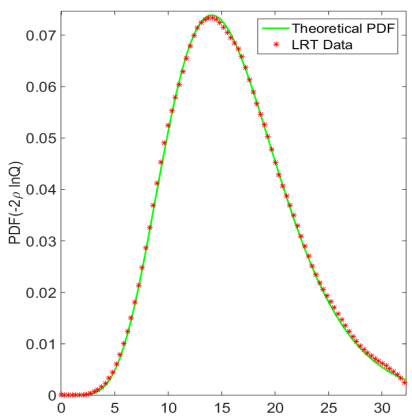

(d) pdf of $\tau_{\text {LRT }}$ for the no-change class
Fig. 6. The LRT statistic for Experiment I. (a) $\tau_{\text {LRT }}$ image for the simulated images shown in Fig. 3. (b) histogram of $\tau_{\text {LRT }}$ characterized by change pixels on the upper tail. (c) change map at the significance level of $1 \%$. Color legend: white, change; gray, no-change.(d) normalized histogram of the LRT statistic for the no-change class together with its asymptotic distribution. The change areas $\mathrm{C}_{1}$ to $\mathrm{C}_{3}$ are presented in Fig. 3(c).

combined tests at significance level of $\alpha_{c}=1 \%$. As discussed above, the upper tail of $\tau_{\mathrm{HLT}}$ and $\tau_{\mathrm{HLT}}^{\prime}$ reflects the higher sensitivity with respect to changes. The two-sided tests on $\tau_{\text {HLT }}$ and $\tau_{\mathrm{HLT}}^{\prime}$ in Fig. 5(a) and 5(b) poorly detect the change areas $\mathrm{C}_{2}$ and $\mathrm{C}_{1}$, respectively, whereas the combined test clearly detects all three change areas as shown in Fig. 5(c). Therefore, we suggest to perform one-sided testing of the maximum HLT statistic rather than two-sided thresholding of either $\tau_{\text {HLT }}$ or $\tau_{\mathrm{HLT}}^{\prime}$. Fig. 5(d) demonstrates the approximate sampling distribution and the histogram of $\tau_{\mathrm{HLT}}$ for the no-change samples. An excellent fit between predicted and observed pdfs is observed by a visual comparison. The average $\mathrm{AD}$ and $\mathrm{KS}$ $p$-values again support the selection of the FS-distribution as a statistical model for the no-change class.

We have tested the LRT statistic given in (27) on this image pair shown in Fig. 3(a) and 3(b). The resulting $\tau_{\text {LRT }}$ is shown in Fig. 6(a). The test statistic $\tau_{\text {LRT }}$ maps all types of change into high values. Thus, the change areas in Fig. 6(a) are bright on a background of dark no-change pixels and correspond to the peaks in the upper tail of the histogram of $\tau_{\text {LRT }}$ in Fig. 6(b), noting that two of the peaks in the histogram are hard to identify. Further, all change pixels are detected by CFARtype thresholding in the upper tail of the distribution in Fig. 6(b). Fig. 6(c) demonstrates the change detection map, where the hypothesis of equal covariance matrices is rejected at $1 \%$ significance level. The histogram of the LRT statistic and its
TABLE III

EXPERIMENT I: CHANGE DETECTION PERFORMANCES

\begin{tabular}{|c|c|c|c|c|}
\hline Specified FAR & Method & Measured FAR & Detection rate & Overall error rate \\
\hline \hline \multirow{2}{*}{$0.5 \%$} & HLT & $0.51 \% \pm 0.02 \%$ & $95.18 \% \pm 0.38 \%$ & $0.72 \% \pm 0.03 \%$ \\
& LRT & $0.52 \% \pm 0.02 \%$ & $86.34 \% \pm 0.07 \%$ & $1.15 \% \pm 0.04 \%$ \\
\hline \multirow{2}{*}{$1 \%$} & HLT & $0.99 \% \pm 0.05 \%$ & $96.70 \% \pm 0.23 \%$ & $0.72 \% \pm 0.04 \%$ \\
& LRT & $1.01 \% \pm 0.04 \%$ & $90.37 \% \pm 0.50 \%$ & $1.17 \% \pm 0.04 \%$ \\
\hline \multirow{2}{*}{$5 \%$} & HLT & $4.54 \% \pm 0.06 \%$ & $99.31 \% \pm 0.10 \%$ & $4.31 \% \pm 0.06 \%$ \\
& LRT & $4.99 \% \pm 0.06 \%$ & $97.50 \% \pm 0.23 \%$ & $4.87 \% \pm 0.06 \%$ \\
\hline \multirow{2}{*}{$10 \%$} & HLT & $9.97 \% \pm 0.10 \%$ & $99.68 \% \pm 0.08 \%$ & $9.96 \% \pm 0.10 \%$ \\
& LRT & $10.01 \% \pm 0.10 \%$ & $98.80 \% \pm 0.22 \%$ & $9.59 \% \pm 0.09 \%$ \\
\hline
\end{tabular}

TABLE IV

CHANGE- TO-BACKGRound RATIO (CBR) OF THE CHANGE AREAS WITH RESPECT TO THE BACKGROUND NO-CHANGE AREA.

\begin{tabular}{|c|c|c|c|}
\hline Test statistic & $\mathrm{C}_{1}$ & $\mathrm{C}_{2}$ & $\mathrm{C}_{3}$ \\
\hline HLT & $5.95 \pm 0.023$ & $3.17 \pm 0.012$ & $36.12 \pm 0.023$ \\
\hline LRT & $5.34 \pm 0.035$ & $2.51 \pm 0.022$ & $9.12 \pm 0.018$ \\
\hline
\end{tabular}

approximate sampling distribution from (29) for the no-change samples are presented in Fig. 6(d).

Table III provides a quantitative evaluation of the change detection performance at four different significance levels or specified FARs, including the measured FAR (i.e., the percentage of no-change pixels erroneously detected as change), the detection rate (i.e., the percentage of change pixels correctly labeled), and the overall error rate (i.e., the percentage of erroneously labeled pixels), for both polarimetric change detectors (HLT and LRT). We provide the values with uncertainty because the data sets are synthesized by means of Monte Carlo simulations. Even though the considered operational setting is unsupervised, such a quantitative accuracy analysis is feasible when we use a simulated data set. As shown in Table III, the HLT statistic achieved higher detection rates and lower overall error rates than the LRT statistic at the specified FARs. The measured FARs for both tests approximately meet the specified FARs.

To perform a thorough analysis, we investigate the contrast between the change areas and the surrounding background. In particular, we investigate the change-to-background ratio (CBR). The parameter is defined as the ratio of the mean value of the change area, $\mu_{c}$, to the mean value of the background no-change region, $\mu_{b}$ :

$$
\mathrm{CBR}=\frac{\mu_{c}}{\mu_{b}} .
$$

The CBR for the three change areas $\mathrm{C}_{1}, \mathrm{C}_{2}$, and $\mathrm{C}_{3}$ with respect to the no-change class is given in Table IV. The HLT produces higher contrast than the LRT for all the change areas in this study, which confirms the change detection results.

To capture the actual performance of the detectors, the receiver operating characteristic (ROC) curves [50] in Fig. 7 present the detection rate as a function of the observed FAR, and not the specified one. This provides a fair comparison, which shows that the proposed HLT statistic outperforms the LRT statisic in this example.

\section{B. Experiment II: Spaceborne Multilook PolSAR Data}

Two single-look complex four-channel quad-pol SAR images acquired by RADARSAT- 2 over an urban area in Suzhou, East China on 9 April 2009 and 15 June 2010 are used for the 


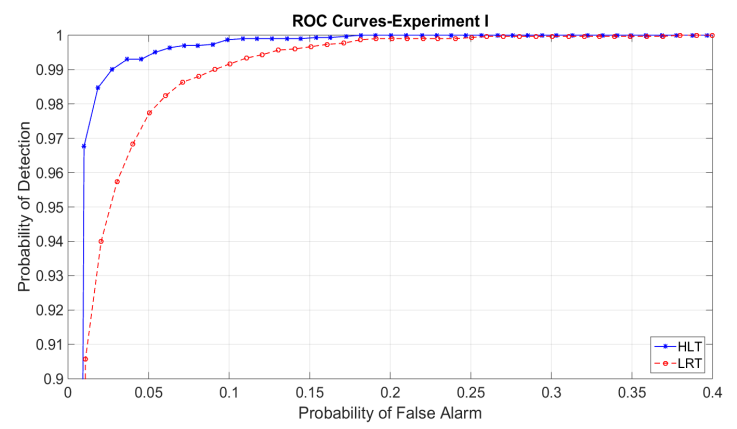

Fig. 7. ROC plot comparison of the two detectors, which plots the probability of detection against the observed probability of false alarm for Experiment I. The HLT detector performs better than the LRT detector.

experiment with real SAR data. The nominal spatial resolution is approximately $5.1 \mathrm{~m} \times 7.7 \mathrm{~m}$ (one-look) in slant range and azimuth directions. Incidence angles range between 38.37 and 39.85 degrees from near range to far range. This area is selected for change detection because of the rapid urban expansion in recent years. First, the two repeat-pass PolSAR images are calibrated and co-registered. Multilooking (with 6 looks in range and 4 looks in azimuth directions) is performed on the SLC data sets, resulting in a pixel dimension of about $30 \mathrm{~m} \times 30 \mathrm{~m}$ and MLC images of size $300 \times 233$ pixels. An enhanced Pauli composite image (red $=\mathrm{HH}-\mathrm{VV}$, green $=\mathrm{HV}$, blue $=\mathrm{HH}+\mathrm{VV}$ ) of each PolSAR image is shown in Fig. 8(a) and 8(b). According to field investigations, two major change classes are labeled as $\mathrm{CH} 1$ and $\mathrm{CH} 2$ in Fig. 8(b). As shown in Fig. 8(e) and 8(f), class $\mathrm{CH} 1$ indicates change due to the appearance of sand panning boats in the river at the time of the second acquisition, whereas class $\mathrm{CH} 2$ indicates the change from water and road to a park containing blue lotus ponds. The reference test map in Fig. 8(d), which contains 3071 nochange test pixels and 1320 change test pixels, was made for the quantitative analysis of change detection results. The regions labeled as change and no-change have been extracted manually. Although data from an urban area may not satisfy the Wishart assumption, the labelled change classes appear in areas that contain distributed scattering, and the experiment should therefore be relevant.

The average ENL of the two images, as discussed in Section III-A, was estimated to 7.2 in a preanalysis of the images. Parameters of the FS distribution are first computed from the average ENL and the polarimetric dimension to model the no-change class. The type of change is assumed unknown before detection, thus we then compute both operators $\tau_{\mathrm{HLT}}=$ $\operatorname{tr}\left(\mathbf{A}^{-1} \mathbf{B}\right)$ and $\tau_{\text {HLT }}^{\prime}=\operatorname{tr}\left(\mathbf{B}^{-1} \mathbf{A}\right)$ over the entire test site, as seen in Fig. 9(a) and 9(b). The linear structures inside red ellipses in Fig. 9(b) correspond to the change areas that $\tau_{\text {HLT }}$ can not identify, whereas those areas are identifiable by the $\tau_{\text {HLT }}^{\prime}$ statistic. The maximum HLT statistic is shown in Fig. 9(c), which maps all change pixels into high values. Finally, the binary decision can be made by CFAR-type thresholding of the combined HLT statistic to get the final change detection map at the significance level of 1\%, as shown in Fig. 9(d). The change map obtained from the two-sided thresholding of $\tau_{\mathrm{HLT}}$ (not shown) does not detect the linear structures in Fig. 9(b).
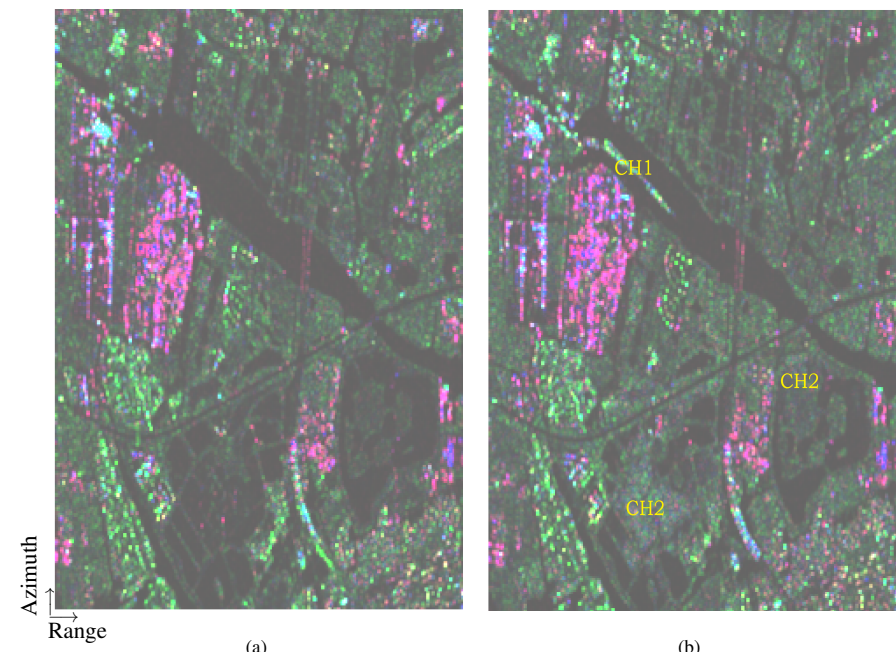

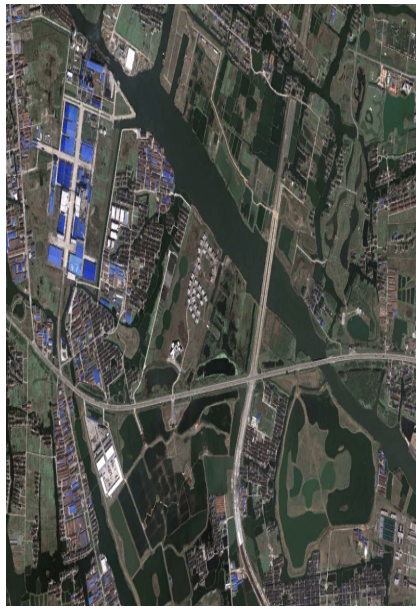

(c)

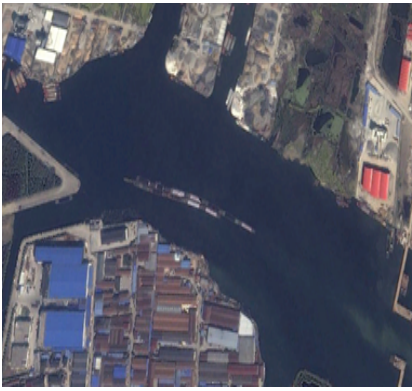

(e) $\mathrm{CH} 1$

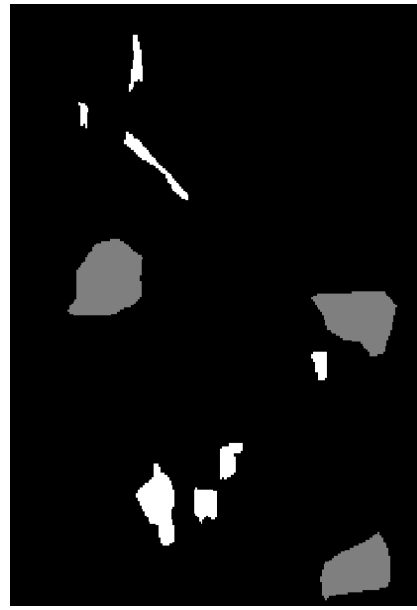

(d)

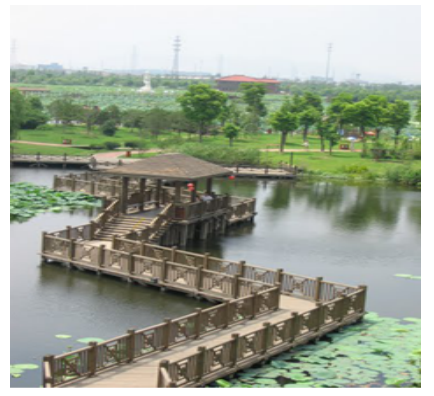

(f) $\mathrm{CH} 2$
Fig. 8. Real experimental data set over Suzhou, East China. (a) and (b) Pauli RGB composite $(\mathrm{R}=\mathrm{HH}-\mathrm{VV}, \mathrm{G}=2 \mathrm{HV}, \mathrm{B}=\mathrm{HH}+\mathrm{VV}$ ) of quad-pol RADARSAT-2 images captured on 9 April, 2009 and on 15 June, 2010, multilooked with 24-looks. (c) corresponding Google Earth image acquired on the first time. (d) reference change map, color legend: white, change; gray, no-change; black, unlabeled pixels. (e) sand panning boats in the river labeled as change class $\mathrm{CH} 1$ in (b). (f) the park constructed on the second time labeled as change class $\mathrm{CH} 2$ in (b).

This confirms again the effectiveness of the one-sided test with the maximum HLT statistic with respect to the two-sided test. It should be noted that there are some changes in Fig. 9(d) that appear in areas containing deterministic scatterers. These areas may departure from the scaled Wishart distribution, but 


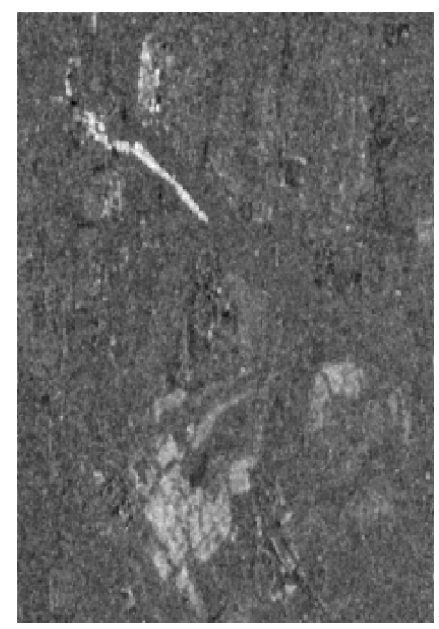

(a) $\log \left(\tau_{\mathrm{HLT}}\right)$

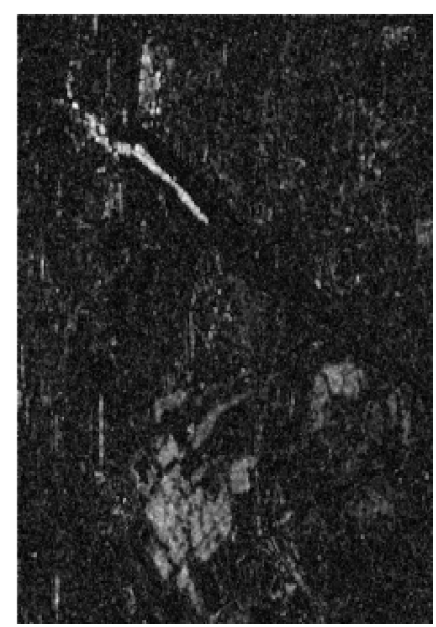

(c) $\log \left(\max \left(\tau_{\mathrm{HLT}}, \tau_{\mathrm{HLT}}^{\prime}\right)\right)$

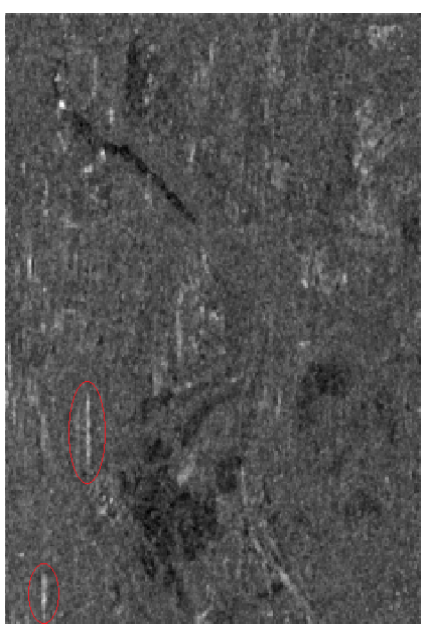

(b) $\log \left(\tau_{\mathrm{HLT}}^{\prime}\right)$

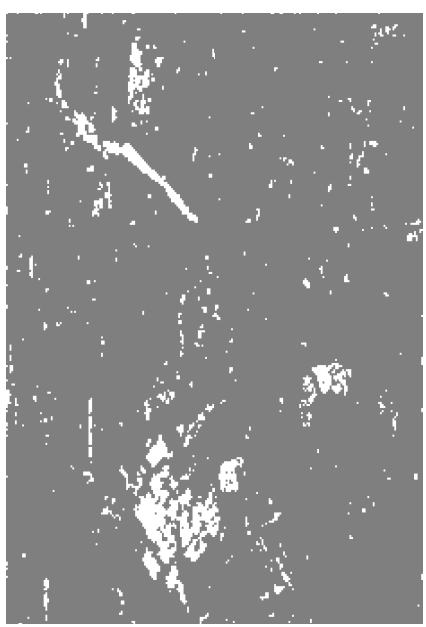

(d) $\max \left(\tau_{\text {HLT }}, \tau_{\text {HLT }}^{\prime}\right) \underset{H_{0}}{\stackrel{H_{1}}{\gtrless}} T_{\text {up }}$

Fig. 9. The HLT statistics for the Experiment II. (a) logarithm of $\tau_{\mathrm{HLT}}=\operatorname{tr}\left(\mathbf{A}^{-1} \mathbf{B}\right)$. (b) logarithm of $\tau_{\mathrm{HLT}}^{\prime}=\operatorname{tr}\left(\mathbf{B}^{-1} \mathbf{A}\right)$. (c) logarithm of $\max \left[\tau_{\mathrm{HLT}}, \tau_{\mathrm{HLT}}^{\prime}\right]$. (d) change detection map at $1 \%$ significance level obtained from the combined test. Color legend: white, change; gray, no-change.

TABLE V

EXPERIMENT II: CHANGE DETECTION PERFORMANCES \begin{tabular}{|l|l|l|l|l|}
\hline Specified FAR & Method & Measured FAR & Detection rate & Overall error rate \\
\hline
\end{tabular}

\begin{tabular}{|c|c|c|c|c|}
\hline \multirow{2}{*}{$0.5 \%$} & HLT & $0.19 \%$ & $79.39 \%$ & $6.33 \%$ \\
& LRT & $0.17 \%$ & $78.63 \%$ & $6.55 \%$ \\
\hline \multirow{2}{*}{$1 \%$} & HLT & $0.30 \%$ & $85.91 \%$ & $4.49 \%$ \\
& LRT & $0.27 \%$ & $83.41 \%$ & $5.10 \%$ \\
\hline \multirow{2}{*}{$5 \%$} & HLT & $1.60 \%$ & $96.06 \%$ & $2.37 \%$ \\
& LRT & $1.55 \%$ & $92.80 \%$ & $3.02 \%$ \\
\hline \multirow{2}{*}{$10 \%$} & HLT & $2.71 \%$ & $97.42 \%$ & $3.41 \%$ \\
& LRT & $2.64 \%$ & $95.23 \%$ & $3.30 \%$ \\
\hline
\end{tabular}

changes were nevertheless observed.

Again, we compare the LRT detector with our proposed algorithm. The test image $\tau_{\text {LRT }}$ is first computed, which is shown in Fig. 10(a). The test statistic shows areas with large change as bright areas and areas with small change as dark areas. The change detection result at $1 \%$ significance level is provided in Fig. 10(b). One strength of the HLT statistics (before combination) is their ability to demonstrate the direction of the change, whereas the LRT does not have

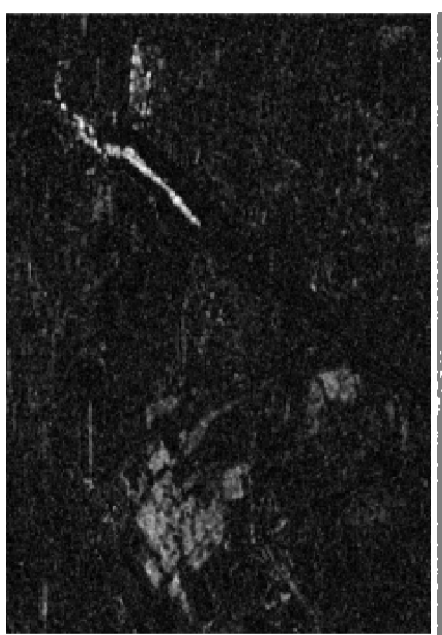

(a) $\tau_{\text {LRT }}$

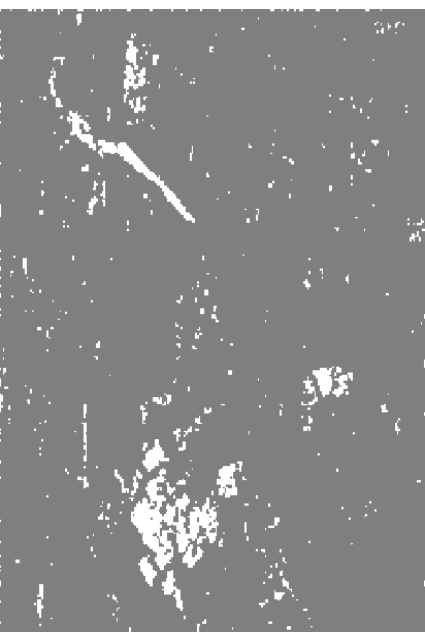

(b) $\tau_{\text {LRT }} \underset{H_{0}}{\stackrel{H_{1}}{\gtrless}} T_{\text {up }}$
Fig. 10. The LRT statistics for the Experiment II. (a) $\tau_{\text {LRT }}$ image. (b) change detection map obtained by rejection of the hypothesis test at $1 \%$ significance level.

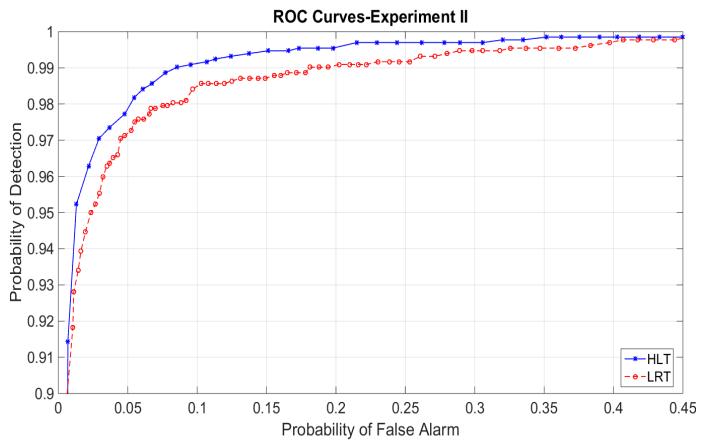

Fig. 11. ROC plot comparison of the two detectors (HLT and LRT) for the Experiment II.

such ability, which could be useful for certain applications where we focus on change from one specific ground cover to another, such as deforestation, flooding, etc.

The final goal of this experiment is to compare the HLT with the conventional LRT detector. Table $\mathrm{V}$ reports the detection rate, the measured FAR, and the overall error rate of the resulting change maps at four different significance levels with respect to the test map in Fig. 8(d). Note that the difference between the specified and measured FARs is because the detection performance is evaluated only on the change and no-change test pixels in Fig. 8(d) by discarding the unlabeled pixels. The maximum HLT statistic obtained higher detection rates and lower overall error rates compared to the LRT detector in this case. To further evaluate the performance of the proposed detector, the ROC curves of the two tests are presented in Fig. 11. The ROC plot of the HLT detector (solid blue line) is above the LRT detector (dashed red line) indicating better detection performance obtained from the HLT statistic in this example. 


\section{DISCUSSION AND CONCLUSION}

We have proposed the complex-kind Hotelling-Lawley trace (HLT) as a test statistic for change detection in multilook PolSAR images. Its sampling distribution has been approximated by an FS distribution, thereby allowing efficient determination of the decision threshold. We have found that the FS distribution can adequately model the sampling distribution under the null hypothesis, which corresponds to no change. This was tested on simulated polarimetric image pairs with different number of looks and polarimetric channels. Using the approximated distribution of the test statistic, change detection can be performed at a specified probability of false alarm, making it suitable for multilook PolSAR images and utilizing the full polarimetric information. The proposed CFAR-type polarimetric change detector has been compared to the classical LRT detector. Experiments with simulated and real PolSAR data sets, for which a reference change map was available, demonstrate the effectiveness of the proposed algorithm. The results in terms of overall error rate, measured FAR, detected changes, and ROCs confirm that the proposed approach can achieve performance very close to the optimal one exhibited by the reference test map. We found from the experiments that the HLT test statistic is more sensitive with respect to changes on the upper tail of the histogram. Therefore, we suggested to calculate both $\operatorname{tr}\left(\mathbf{A}^{-1} \mathbf{B}\right)$ and $\operatorname{tr}\left(\mathbf{B}^{-1} \mathbf{A}\right)$ and apply the CFARtype thresholding to their maximum, rather than the two-sided thresholding on one of them. Experiments confirm that better detection performance is achieved by one-sided testing of the maximum HLT statistic with respect to the two-sided test.

The main advantages of the proposed approach are: 1) its mathematical simple characteristics that can be seen as a matrix-variate extension of the intensity ratio method for the PolSAR change detection; 2) the utility of the FS distribution as a flexible model for approximation of the sampling distribution of the test statistic; and 3) that the method is totally unsupervised, which is an important aspect since ground truth data are in many cases not available. The success of the method hinges on accurate estimation of the ENL, for which we use a state-of-the-art method. The method could be extended to a non-Wishart model, which incorporates textural variation. However, the gain in using a more flexible model may easily be lost in the estimation error of additional parameters. Therefore, it is not certain that this will improve the change detection result, unless the texture is strong. On the other hand, separation of changes in intensity from changes in polarimetry requires a refined approach.

Moreover, the proposed method is aimed at extracting, from a multitemporal pair of PolSAR images, a scalar feature that accurately discriminates changed and unchanged areas. Further possible extensions of this work could be the combination of this feature with automatic thresholding techniques, based for example on generalizations of the K\&I approach [3], or with contextual unsupervised classification methods, based for instance on Markov random fields. The latter extension would be especially useful in minimizing the impact of speckle on the resulting change map [51].

\section{ACKNOWLEDGMENT}

RADARSAT-2 Data and Products (C)MacDonald, Dettwiler and Associates Ltd. (2011)-All Rights Reserved. RADARSAT is an official mark of the Canadian Space Agency. The authors would like to thank Meng Liu, and the Institute of Remote Sensing and Digital Earth, Chinese Academy of Sciences for providing the data set. We also acknowledge the anonymous reviewers for their suggestions that have helped to improve the paper.

\section{REFERENCES}

[1] E. J. Rignot and J. J. Van Zyl, "Change detection techniques for ERS1 SAR data," IEEE Trans. Geosci. Remote Sens., vol. 31, no. 4, pp. 896-906, Jul. 1993.

[2] Y. Bazi, L. Bruzzone, and F. Melgani, "An unsupervised approach based on the generalized Gaussian model to automatic change detection in multitemporal SAR images," IEEE Trans. Geosci. Remote Sens., vol. 43, no. 4, pp. 874-887, Apr. 2005.

[3] G. Moser and S. B. Serpico, "Generalized minimum-error thresholding for unsupervised change detection from SAR amplitude imagery," IEEE Trans. Geosci. Remote Sens., vol. 44, no. 10, pp. 2972-2982, Oct. 2006.

[4] J. Inglada and G. Mercier, "A new statistical similarity measure for change detection in multitemporal SAR images and its extension to multiscale change analysis," IEEE Trans. Geosci. Remote Sens., vol. 45, no. 5, pp. 1432-1445, May 2007.

[5] G. Moser and S. B. Serpico, "Unsupervised change detection from multichannel SAR data by Markovian data fusion," IEEE Trans. Geosci. Remote Sens., vol. 47, no. 7, pp. 2114-2128, Apr. 2009.

[6] V. Akbari, A. Doulgeris, and T. Eltoft, "Monitoring glacier changes using multitemporal multipolarization SAR images," IEEE Trans. Geosci. Remote Sens., vol. 52, no. 6, pp. 3729-3741, Jun. 2014.

[7] L. M. Novak, "Coherent change detection for multi-polarization SAR," Conf. Rec. 39th Asilomar Conf. Signals, Syst. Comput., Pacific Grove, CA, Oct. 28- Nov. 1, 2005, pp. 568-573.

[8] M. Gong, Z. Zhou, and J. Ma, "Change detection in synthetic aperture radar images based on image fusion and fuzzy clustering," IEEE Trans. Image Process., vol. 21, no. 4, pp. 2141-2151, Apr. 2012.

[9] C. Oliver and S. Quegan, Understanding Synthetic Aperture Radar Images, 2nd ed. Raleigh, NC: SciTech Publishing, 2004.

[10] R. Touzi, A. Lopes, and P. Bousquet, "A statistical and geometrical edge detector for SAR images," IEEE Trans. Geosci. Remote Sens., vol. 26, no. 6, pp. 764-773, May 1988.

[11] C. Carincotte, S. Derrode, and S. Bourennane, "Unsupervised change detection on SAR images using fuzzy hidden Markov chains," IEEE Trans. Geosci. Remote Sens., vol. 44, no. 2, pp. 432-441, Feb. 2006.

[12] R. G. Caves and S. Quegan, "Segmentation based change detection in ERS-1 SAR images," in Proc. IEEE IGARSS, Pasadena, CA, Aug. 8-12, 1994, vol. 4, pp. 2149-2151.

[13] P. Lombardo and T. M. Pellizzeri, "Maximum likelihood signal processing techniques to detect a step pattern of change in multitemporal SAR images," IEEE Trans. Geosci. Remote Sens., vol. 40, no. 4, pp. 853-870, Apr. 2002.

[14] K. Conradsen, A. A. Nielsen, J. Schou, and H. Skriver, "A test statistic in the complex Wishart distribution and its application to change detection in polarimetric SAR data," IEEE Trans. Geosci. Remote Sens., vol. 41, no. 1, pp. 4-19, Jan. 2003.

[15] P. R. Kersten, J. S. Lee, and T. L. Ainsworth, "A comparison of change detection statistics in POLSAR images," in Proc. IEEE IGARSS, Seoul, South Korea, Jul. 25-29, 2005, vol. 7, pp. 4836-4839.

[16] E. Erten, A. Reigber, L. Ferro-Famil, and O. Hellwich, "A new coherent similarity measure for temporal multichannel scene characterization," IEEE Trans. Geosci. Remote Sens., vol. 50, no. 7, pp. 2839-2851, Jul. 2012.

[17] A. Marino, S. Cloude, and J. Sanchez-Lopez, "A new polarimetric change detector in radar imagery," IEEE Trans. Geosci. Remote Sens. vol. 51, no. 5, pp. 2986-3000, May 2013.

[18] M. Liu, H. Zhang, C. Wang, and F. Wu, "Change detection of multilook polarimetric SAR images using heterogeneous clutter models," IEEE Trans. Geosci. Remote Sens., vol. 52, no. 12, pp. 7483-7494, Dec. 2014.

[19] V. Akbari, S. Anfinsen, A. Doulgeris, and T. Eltoft, "A change detector for polarimetric SAR data based on the relaxed Wishart distribution," in Proc. IEEE IGARSS, Milan, Italy, Jul. 26-31, 2015, pp. 3806-3809. 
[20] K. Conradsen, A. A. Nielsen, and H. Skriver, "Change detection in polarimetric SAR data over several time points," in Proc. IEEE IGARSS, Quebec City, QC, Jul. 13-18, 2014, pp. 4540-4543.

[21] A. A. Nielsen, K. Conradsen, and H. Skriver, "Change detection in full and dual polarization, single-and multifrequency SAR data," IEEE Sel. Topics Appl. Earth Obs. Remote Sens., vol. 8, no. 8, pp. 4041-4048, Aug. 2015.

[22] G. Liu, L. Jiao, F. Liu, H. Zhong, and S. Wang, "A new patch based change detector for polarimetric SAR data," Pattern Recognit., vol. 48, no. 3, pp. 685-695, Mar. 2015.

[23] Z. Qi, A. G.-O. Yeh, X. Li, S. Xian, and X. Zhang, "Monthly short-term detection of land development using RADARSAT-2 polarimetric SAR imagery," Remote Sens. of Environ., vol. 164, pp. 179-196, Jul. 2015.

[24] Z. Qi, A. G.-O. Yeh, X. Li, and X. Zhang, "A three-component method for timely detection of land cover changes using polarimetric SAR images," ISPRS J. Photogramm. Remote Sens., vol. 107, pp. 3-21, Sep. 2015.

[25] T. T. Lê, A. M. Atto, E. Trouvé, A. Solikhin, and V. Pinel, "Change detection matrix for multitemporal filtering and change analysis of SAR and PolSAR image time series," ISPRS J. Photogramm. Remote Sens., vol. 107, pp. 64-76, Sep. 2015.

[26] J. R. Bunch and R. D. Fierro, "A constant-false-alarm-rate algorithm," Linear Algebra Appl., vol. 172, pp. 231-241, Jul. 1992.

[27] N. Otsu, "A threshold selection method from gray-evel histograms," IEEE Trans. Syst. Man and Cybernetics, vol. 9, no. 1, pp. 62-66, Jan. 1979.

[28] J. Kittler and J. Illingworth, "Minimum error thresholding," Pattern Recognit., vol. 19, no. 1, pp. 41-47, 1986.

[29] J. N. Kapur, P. K. Sahoo, and W. A. K. C., "A new method for gray-level picture thresholding using the entropy of the histogram," Comput. Vis. Graph. Image Process., vol. 29, no. 3, pp. 273-285, Mar. 1985.

[30] Z. Liu, J. D. Jiang and H. Feng, "A novel fuzzy classification entropy approach to image thresholding," Pattern Recognit. Lett., vol. 27, no. 16, pp. 1968-1975, Dec. 2006.

[31] V. Akbari, A. P. Doulgeris, S. Anfinsen, and T. Eltoft, "The HotellingLawley trace statistic for change detection in polarimetric SAR data under the complex Wishart distribution," in Proc. IEEE IGARSS, Melbourne, Australia, Jul. 22-26, 2013, pp. 4162-4165.

[32] K. C. S. Pillai and P. Samson, "On Hotelling's Generalization of T2," Biometrika, vol. 46, no. 1/2, pp. 160-168, Jun. 1959.

[33] D. T. Hughes and J. G. Saw, "Approximating the percentage points of Hotelling's generalized $T_{0}^{2}$ statistic," Biometrika, vol. 59, no. 1, pp. 224-226, Apr. 1972.

[34] J. J. McKeon, " $F$ Approximations to the distribution of Hotelling's $T_{0}^{2}$," Biometrika, vol. 61, no. 2, pp. 381-383, Aug. 1974

[35] S. N. Anfinsen, A. P. Doulgeris, and T. Eltoft, "Estimation of the equivalent number of looks in polarimetric synthetic aperture radar imagery," IEEE Trans. Geosci. Remote Sens., vol. 47, no. 11, pp. 37953809, Nov. 2009.

[36] J.-S. Lee and E. Pottier, Polarimetric Radar Imaging: From Basics to Applications, Optical Science and Engineering, Boca Raton, FL: CRC Press, 2009.

[37] A. van den Bos, "The multivariate complex normal distribution -a generalization," IEEE Trans. Inf. Theory, vol. 41, no. 2, pp. 537-539, Mar. 1995.

[38] N. R. Goodman, "Statistical analysis based on a certain multivariate complex Gaussian distribution (an introduction)," Ann. Math. Statist., vol. 34, no. 1, pp. 152-177, Mar. 1963.

[39] J. Lee, M. Grunes, and R. Kwok, "Classification of multi-look polarimetric SAR imagery based on the complex Wishart distribution," Int. J. Remote Sens., vol. 15, no. 11, pp. 2299-2311, Jul. 1994.

[40] S. N. Anfinsen, A. P. Doulgeris, and T. Eltoft, "Goodness-of-fit tests for multilook polarimetric radar data based on the Mellin transform," IEEE Trans. Geosci. Remote Sens., vol. 49, no. 7, pp. 2764-2781, Jul. 2011.

[41] S. N. Anfinsen, "Statistical analysis of multilook polarimetric radar images with the Mellin transform," Ph.D. dissertation, University of Troms $\varnothing$, Norway, May 2010.

[42] F. Galland, J.-M. Nicolas, H. Sportouche, M. Roche, F. Tupin, and P. Refregier, "Unsupervised synthetic aperture radar image segmentation using Fisher distributions," IEEE Trans. Geosci. Remote Sens., vol. 47, no. 8, pp. 2966-2972, Aug. 2009.

[43] L. Bombrun, G. Vasile, M. Gay, and F. Totir, "Hierarchical segmentation of polarimetric SAR images using heterogeneous clutter models," IEEE Trans. Geosci. Remote Sens., vol. 49, no. 2, pp. 726-737, Feb. 2011.

[44] P. Graczyk, G. Letac, and H. Massam, "The complex Wishart distribution and the symmetric group," Ann. Statist., vol. 31, no. 1, pp. 287-309, Feb. 2003.
[45] W. C. Parr and W. R. Schucany, "Minimum distance estimation and components of goodness-of-fit statistics," J. Royal Statist. Soc., ser. B, vol. 44, no. 2, pp. 178-189, 1982.

[46] A. Alonso-González, "Multidimensional and temporal SAR data representation and processing based on binary partition trees," $\mathrm{Ph} . \mathrm{D}$ dissertation, Universitat Politécnica de Catalunya, Barcelona, Spain, Jun. 2014.

[47] A. Alonso-González, T. Jagdhuber, and I. Hajnsek, "Exploitation of agricultural polarimetric SAR time series with binary partition trees," in Proc. ESA PolInSAR, Frascati, Italy, Jan. 26-30, 2015, vol. ESA SP$729,8 \mathrm{pp}$.

[48] A. P. Doulgeris, S. Anfinsen, and T. Eltoft, "Classification with a nonGaussian model for PolSAR data," IEEE Trans. Geosci. Remote Sens., vol. 46, no. 10, pp. 2999-3009, Oct. 2008.

[49] R. D'Agostino and M. A. Stephens, Goodness-of-Fit Techniques, Marcel Dekker, Inc., New York, NY, 1986.

[50] C. E. Metz, "Basic principles of ROC analysis," Seminars Nucl. Med., vol. 8, no. 4., pp. 283-298, Oct. 1978,

[51] V. Akbari, A. Doulgeris, G. Moser, T. Eltoft, S. Anfinsen, and S. Serpico, "A textural-contextual model for unsupervised segmentation of multipolarization synthetic aperture radar images," IEEE Trans. Geosci. Remote Sens., vol. 51, no. 4, pp. 2442-2453, Apr. 2013.

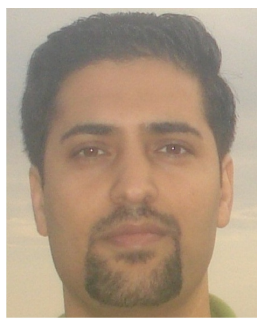

Vahid Akbari (S'10-M'14) received the M.Sc. degree in remote sensing (summa cum laude) from the University of Tehran, Iran, in 2009 and the Ph.D. degree in physics from the University of Troms $\varnothing$, Troms $\varnothing$, Norway, in 2013.

$\mathrm{He}$ is continuing his research in radar remote sensing as a Postdoctoral Research Fellow at the Department of Physics and Technology, University of Troms $\varnothing-$ The Arctic University of Norway. His main research interests statistical modeling for segmentation, change detection, and target detection in polarimetric synthetic aperture radar images.

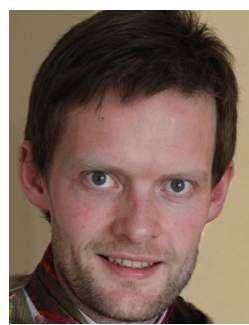

Stian N. Anfinsen (S'06-M'10) eceived the Cand. Mag. (B.Sc.), Cand. Scient. (M.Phil.) and Ph.D. degrees in physics from the University of Troms $\varnothing$, Troms $\varnothing$, Norway, in 1997, 2000 and 2010, respectively. He received the M.Sc. in communications, Control and Digital Signal Processing with distinction from the University of Strathclyde, Glasgow, U.K. in 1998

He was with satellite ground station system provider Kongsberg Spacetec in Troms $\varnothing$ from 2001 to 2005. He has been with University of Troms $\varnothing-$ The Arctic University of Norway, Department of Physics and Technology, since 2005 as a Research Fellow, Postdoctoral Research Fellow, and since 2014 as an Associate Professor. His current research focuses on statistical modelling, parameter estimation, classification, change detection and target detection in polarimetric synthetic aperture radar images, and remote sensing of forest and vegetation. He has been recognized as the 2012 Best Reviewer of the IEEE Journal of Selected Topics in Applied Earth Observations and Remote Sensing.

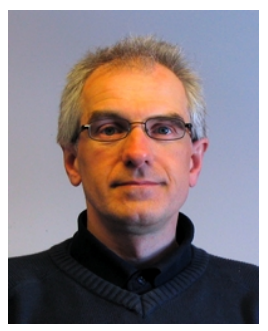

Anthony P. Doulgeris received the B.Sc. degree in physics from the Australian National University, Canberra, Australia, in 1988, and the M.Sc. degree and $\mathrm{Ph} . \mathrm{D}$. degree in physics from the Department of Physics and Technology, University of Troms $\varnothing$, Troms $\varnothing$, Norway, in 2006, and 2011, respectively.

He joined the Department of Physics and Technology at the University of Troms $\varnothing$ - The Arctic University of Norway, in 2007 and is currently an Associate Professor in applied Earth observation. His research interests focus on investigating remote sensing, pattern recognition and multidimensional statistical modelling, in particular with polarimetric synthetic aperture radar images. 


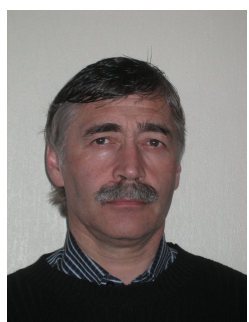

Torbjørn Eltoft (M'92) the degrees of Cand. Real. (M.Sc.) and Dr. Scient. (Ph.D.) from the University of Troms $\varnothing$, Troms $\varnothing$, Norway, in 1981 and 1984, respectively. In 1988 he joined the Faculty of Science and Technology, University of Troms $\varnothing$ - The Arctic University of Norway, where he was Head of the Department of Physics and Technology from 2013 to 2015, and is currently leading CIRFA, a new Centre for Research-based Innovation at the University of Troms $\emptyset$. He is a Professor in electrical engineering, and holds a position as Adjunct Professor at the Northern Research Institute (Norut), Troms $\varnothing$. His research interests include multi-dimensional signal and image analysis with application in radar remote sensing, statistical models, neural networks, and machine learning.

Prof. Eltoft has a significant publication record in the area of research signal processing and remote sensing, $\mathrm{He}$ was the recipient of the year 2000 Outstanding Paper Award in Neural Networks awarded by IEEE Neural Networks Council, and of the Honorable Mention for the 2003 Pattern Recognition Journal Best Paper Award. He has supervised 15 PhD- and numerous of Master-students.

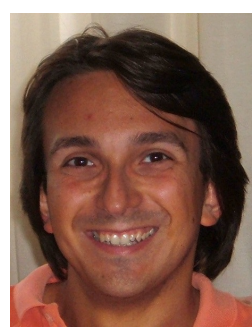

Gabriele Moser (S'03-M'05) received the M.Sc. degree in telecommunications engineering (summa cum laude) and the Ph.D. degree in space sciences and engineering from the University of Genoa, Genoa, Italy, in 2001 and 2005, respectively.

Since 2014, he has been an Associate Professor of telecommunications with the University of Genoa. Since 2001, he has cooperated with the Signal Processing and Telecommunications Research Laboratory, University of Genoa. From January to March 2004, he was a visiting student at the Institut National de Recherche en Informatique et en Automatique (INRIA), Sophia Antipolis, France. He has been a reviewer for several international journals. His research activity is focused on the development of image-processing and pattern-recognition methodologies for remote sensing data interpretation. His current research interests include contextual classification, multitemporal image classification and change detection, SAR data analysis, hyperspectral image classification, and geo/biophysical parameter estimation.

Dr. Moser has been an Associate Editor of the IEEE Geoscience and Remote Sensing Letters and Pattern Recognition Letters since 2008 and 2011, respectively.

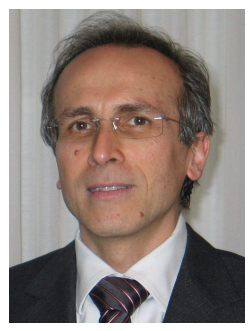

Sebastiano B. Serpico (M'87-SM'00-F'08) received the M.Sc. degree in electronic engineering and the Ph.D. degree from the University of Genoa, Genoa, Italy, in 1982 and 1989, respectively.

$\mathrm{He}$ is a Full Professor of telecommunications with the Polytechnic School, University of Genoa, where he is the Head of the Image Processing and Pattern Recognition for Remote Sensing (IPRS) Laboratory, Department of Electrical, Electronic, Telecommunications Engineering and Naval Architecture (DITEN). He is the Chairman of the Institute of Advanced Studies in Information and Communication Technologies (ISICT) $\mathrm{He}$ has been the project manager of numerous research contracts and an evaluator of project proposals for various programs of the European Union. $\mathrm{He}$ is the author or coauthor of more than 200 scientific articles published in journals and conference proceedings. His current research interests include pattern recognition for remote sensing images and for biomedical images.

Prof. Serpico has been an Associate Editor of the IEEE TRANSACTIONS ON GEOSCIENCE AND REMOTE SENSING (TGRS) since 2001. He was a Guest Editor of two special issues of TGRS on "Analysis of Hyperspectral Image Data" (July 2001) and "Advances in Techniques for the Analysis of Remote Sensing Data" (March 2005). From 1998 to 2002, he was the Chairman of a SPIE/EUROPTO series of conferences on signal and image processing for remote sensing. He was the recipient of the Best Paper Award at the 2010 IEEE Workshop on Hyperspectral Image and Signal Processing. 